\title{
Light-fuelled dissipative replication and selection in adaptive biomimetic chemical networks
}

\author{
Éva Bartus ${ }^{1,2}$, Beáta $\mathrm{Mag}^{1}$, Áron Bajcsi ${ }^{1}$, Attila Tököli ${ }^{1}$, Gábor Kecskeméti ${ }^{1}$, Edit Wéber ${ }^{1}$, \\ Zoltán Kele $^{1}$, Gabriel Fenteany ${ }^{1,3}$, Tamás A. Martinek ${ }^{1,2 *}$
}

\author{
Affiliations: \\ ${ }^{1}$ Department of Medical Chemistry, University of Szeged, Dóm tér 8, H-6720 Szeged, \\ Hungary \\ ${ }^{2}$ MTA-SZTE Biomimetic Systems Research Group, University of Szeged, Dóm tér 8, H- \\ 6720 Szeged, Hungary \\ ${ }^{3}$ Institute of Genetics, Biological Research Centre, Temesvári krt. 62, H-6726 Szeged, \\ Hungary \\ *Corresponding author. Email: martinek.tamas@med.u-szeged.hu
}

Evolvability of chemical replicator systems requires non-equilibrium energy dissipation, effective decomposition pathways and transfer of structural information in the autocatalytic cycles. We engineered a chemical network with peptidic foldamer components, which displays dissipative sequence-dependent replication and replicator decomposition fuelled by UV light. The light-harvesting formation-recombination cycle of thiyl radicals was coupled with the molecular recognition steps in the replication cycles. Thiyl radical-mediated chain reaction was responsible for the replicator breakdown. The competing and kinetically asymmetric replication and decomposition processes led to light intensity-dependent selection, which differs from the equilibrium composition. The results contribute to the development of chemically evolvable dissipative replicator systems.

\section{Introduction}

Chemical networks mimicking evolution need to operate dynamically far from equilibrium ${ }^{1}$, which is essential for their open-ended adaptive behaviour. The dynamic kinetic stability in such systems results from the balance between the asymmetric formation and destruction processes driven by the dissipation of energy harvested from the environment ${ }^{2-4}$. Besides metabolism and compartmentalisation, dissipative replication and selection are crucial components for modelling chemical evolution. Non-equilibrium dynamics occur in dissipative chemical networks ${ }^{2}, 5,6$, which rely on an energy-harvesting catalytic cycle covering the entropy production of the system. Engineered dissipative systems ${ }^{3,7}$ attain off-equilibrium states present in the non-covalent assembly of the building blocks ${ }^{8-10}$. The dissipative selforganisation of material can be a source of structural complexity ${ }^{11-13}$ linked to the emergence of life.

Chemical ${ }^{13}$ or light energy ${ }^{14,15}$ can promote chemical replication by producing precursors in a protometabolic manner. However, driving replication and replicator decomposition simultaneously with external energy is a current challenge. Our goal was to find a feasible energy-harvesting covalent chemistry that drives both synthetic and breakdown processes asymmetrically depending on the intensity of the energy influx. UV-induced photochemical rearrangement reactions can be carried out with aliphatic disulfides in the organic liquid 
phase $^{16,17}$ and in aqueous medium. ${ }^{18,19}$ Thorough kinetic analysis established that both UVAand UVB-induced photolysis produces thiyl radicals, which participate in a diffusioncontrolled chain reaction. Depending on the steric accessibility, the exchange proceeds through radical substitution or recombination. Strikingly, anomalously high reaction rates were obtained for the bulky iso-butyl substituent. ${ }^{16}$ The authors assumed in this case that the reaction is not governed by the diffusion-controlled collision of the thiyl radicals. These seminal findings suggest that photochemical disulfide rearrangement facilitates both diffusioncontrolled and proximity-controlled mechanisms in a substituent-dependent manner. Since these kinetically asymmetric mechanisms are of different order functions of light intensity (see below), they promise an energy-dependent response, potentially paving the way toward dissipative behaviour.

We set out to test the photochemical disulfide rearrangement with a diverse population of helical foldamers as substituents in the aqueous medium. We hypothesised that preferential binding between the foldamer chains could exert side chain-dependent proximity control over photochemical exchange. In contrast, non-interacting segments should readily enter the diffusion-governed chain reaction. The competition between the two processes was expected to yield a UVA light intensity-dependent composition without damaging the peptidic chains. Designed helical peptides can transfer structural information in autocatalytic processes ${ }^{20,21}$, and exponential replication has been achieved for peptidic helices ${ }^{22}$. Helical foldamers are excellent models because the folding can be readily controlled to generate the biomimetic recognition surface ${ }^{23,24}$. Moreover, short peptidomimetic foldamers tend to self-associate in solution $^{25}$, which is an advantageous feature in terms of proximity control. Seeking potential autocatalytic phenomena, we investigated the influence of the foldamer-foldamer interactions on energy-harvesting thiyl chemistry.

Here, we show that UVA light-fuelled disulfide rearrangement can drive a chemical network to off-equilibrium states in a dissipative manner. We found that cross-/autocatalytic templating plays a dominant role in the exchange processes facilitating replication. A competing breakdown mechanism influenced the replicator concentration and thereby laid the foundation for the adaptive selection phenomenon.

\section{Results}

\section{Construction of the foldamer-based photochemical disulfide rearrangement network}

We constructed the reaction network using hexameric $\beta$-peptide foldamers designed to fold into compact helical structures (Fig. 1a). The sequences contained two variable positions (Xaa ${ }^{1}$ and $\mathrm{Xaa}^{2}$ ), where proteinogenic side chains were incorporated. These can successfully probe protein surfaces $^{23}$, and the ordered secondary structure promotes self-association ${ }^{25}$. We chose 12 different side-chain combinations based on their tendency to self-associate and the ability to bind to hydrophobic patches ${ }^{26}$. We did not pursue other designs to keep the complexity of the sequences at a low level. Cys residues were attached to the C-terminus, which allowed for disulfide linkage between foldamer segments (dimers, MSSM) and glutathione (monomers, MSSG). This setup yielded 78 different dimers and 12 glutathione-protected monomers (Fig. 1a, Supplementary Table 1). 
Before the photochemical experiments, the sequence-dependent association tendency of the foldamer segments was validated in an aqueous medium by relaxing the system to equilibrium with the dynamic covalent thiolate mechanism (Supplementary Fig. 1). In equilibrium, the product distribution was biased toward the hydrophobic dimers indicating sequence-dependent association. For the photochemical exchange experiments, free thiol groups were not present in the system, and the $\mathrm{pH}$ was set to 7.0 to eliminate light-independent thiolate-mediated disulfide exchange. The disulfide rearrangement reactions starting from the mixture of pure MSSG sequences were driven by UVA irradiation $(365 \mathrm{~nm})$ at constant temperature $(303 \mathrm{~K})$.

Figure 1. General sequences of the components and the steps of the photochemical disulfide-exchange mechanisms. (a) MSSG: glutathione-protected monomers, MSSM: disulfide-linked dimers. SG indicates the glutathione ( $\gamma$-L-glutamyl-L-cysteinyl-glycine) and ACHC stands for $1 S, 2 S$-2-aminocyclohexanecarboxylic acid, which promotes helical folding. In the highlighted positions $\mathrm{Xaa}^{1}$ and $\mathrm{Xaa}^{2}, \beta^{3}$-amino acids with proteinogenic side chains were incorporated in the following combinations: IF, KW, LW, QW, RW, RF, SW, TW, VW, WF, WW and YF. One-letter codes correspond to the side chains in the standard $\alpha$-amino acid notation. (b) Diffusion-controlled radical chain-reaction mechanism as described in the literature for the photochemical disulfide exchange ${ }^{16}$. The separate initiation ([1a] and [2a]), termination ([1b] and [2b]) and chain propagation steps ([3a,b] and [4a,b]) are indicated with the back-and-forth arrows, which do not refer to any preequilibrium or microscopic reversibility. (c) The hypothesised proximity-controlled reaction pathway with the foldamer association preequilibrium [5]. The intracomplex steps producing MSSM can be radical substitution ([7]) and concerted metathesis [9].

\section{Competing diffusion- and proximity-control predicts light intensity-dependent steady- state composition}

The light-induced thiyl radicals participate in a chain reaction as established in the literature ${ }^{16}$, 19 (Fig. 1b, [1]-[4]). In the absence of steric inhibition and preferential binding, the predominant exchange route is diffusion-controlled radical substitution (Fig. 1b, [3] and [4]). Based on the preceding literature observation, the rate equations for dimer synthesis [3a] $\left(v_{s, c h}\right)$ 
and breakdown [3b] $\left(v_{b}\right)$ can be computed (eqs. (1) and (2)). The detailed calculations are provided in the Supplementary text.

$$
\begin{aligned}
v_{s, c h} & =s_{c h} \sqrt{I}[M S S G]^{1.5} \\
v_{b} & =b \sqrt{I}[M S S M]
\end{aligned}
$$

The terms $s_{c h}$ and $b$ represent the corresponding rate constants, and $I$ designates light intensity. The central hypothesis of this work is that preferential binding (Fig. 1c, [5]) facilitates the proximity-controlled exchange pathways. In this case, the light-induced homolytic cleavage [6] and the subsequent radical substitution [7] occur within the foldameric complexes. We cannot rule out a priori a concerted metathesis with coincident absorption of two photons (Fig. $1 \mathrm{c},[8]$ and [9]). In this case, the cross section of the interaction is not decreased by non-linear two-photon absorption effects because the two disulfides are separately excited. The corresponding rate equations for MSSM formation via [7] $\left(v_{s, p l}\right)$ and [9] $\left(v_{s, p 2}\right)$ can be derived through a binding preequilibrium and the rate-limiting intracomplex steps (eqs. (3) and (4)). See Supplementary text for the detailed computation.

$$
\begin{gathered}
v_{s, p 1}=s_{p 1} I[M S S G]^{2} \\
v_{s, p 2}=s_{p 2} I^{2}[M S S G]^{2}
\end{gathered}
$$

The proximity-controlled radical substitution and metathesis rate constants are $s_{p 1}$ and $s_{p 2}$, respectively. The steady state concentration of the dimers $\left([\mathrm{MSSM}]_{\mathrm{ss}}\right)$ can be readily expressed using rearranged terms from eqs. (1) - (4) to yield eq. (5).

$$
[\mathrm{MSSM}]_{s s}=\frac{s_{p 1} \sqrt{I}[\mathrm{MSSG}]^{2}+s_{p 2} I^{1.5}[\mathrm{MSSG}]^{2}+s_{c h}[\mathrm{MSSG}]^{1.5}}{b}
$$

This result predicts light intensity-dependent steady-state composition for the system if (i) dynamic kinetic stability can be attained and (ii) the foldamer association facilitates proximitycontrolled synthesis.

\section{Light intensity- and substituent-dependent kinetic asymmetry in the photochemical foldamer-disulfide exchange}

We tested the behaviour of the reaction network upon exposure to UVA irradiation. The power density was increased linearly in four steps up to the maximum value available in our setup $\left(5.10 \mathrm{~mW} \mathrm{~cm}^{-2}\right)$. Without irradiation and at $25 \%$ light intensity, we could not detect dimer formation. At 50\% and above, dimers were observed, and the system attained dynamic kinetic stability at low conversions (without monomer depletion) in $5 \mathrm{~h}$ (Fig. 2a). UVA-induced degradation of the peptidic sequences was not detected. These observations support that dynamic kinetic stability was attained through competition between the breakdown and synthetic processes.

The increasing light intensity caused a non-linear and sequence-dependent growth of the steady-state dimer population (Fig. 2b). We calculated the light intensity-dependent 
amplifications for the dimers upon elevating the power density from $50 \%$ to $100 \%$ (Fig. 2c). For the set of the ten most populated sequences relative to the complete set of dimers, enrichment of aromatic and aliphatic hydrophobic side chains was detected (Fig. 2d). In parallel, depletion of polar and cationic residues was measured at the peripheral positions (1 and 1'). These findings confirmed the predicted light intensity-dependent steady-state concentrations. Thus, we can conclude that proximity-controlled synthesis is present in the system, with efficiency dependent on sequence.

The steady-state concentrations steeply converge to zero with decreasing light intensity for all sequences (Fig. 2b). This result strongly suggests that the diffusion-controlled radical substitution (Fig. 1b, [3a]) $\left(v_{s, c h}\right)$ has no detectable contribution to the dimer synthesis; that is, the light-independent term is negligible in the numerator of eq. (5). Dimer synthesis, therefore, proceeds predominantly via the proximity-controlled pathways, whereas breakdown to monomers occurs through diffusion-controlled radical substitution. Thus, an energy influxdependent kinetic asymmetry determines the behaviour of the system.

Figure 2. Light intensity- and substituent-dependent concentrations. (a) Time- and light intensity-dependent concentration of a representative dimer (WF-YF) obtained at power densities of $2.55 \mathrm{~mW} \mathrm{~cm}^{-2}$ (diamonds), $3.80 \mathrm{~mW} \mathrm{~cm}^{-2}$ (triangles), $5.10 \mathrm{~mW} \mathrm{~cm}^{-2}$ (circles). (b) Light intensity-dependent steady state concentrations for WF-YF (circles). (c) Light intensitydependent amplifications obtained upon increasing power density from 50\% to $100 \%$ with the ten most amplified sequences indicated (inset). (d) Side-chain enrichments in the peripheral (1, $\left.1^{\prime}\right)$ and the central $\left(2,2^{\prime}\right)$ positions for the ten most amplified dimers (n.a. stands for 'not applicable'). 
Next, we attempted to fit the dynamic model defined by the rate equations (2)-(4) to the complete time- and light intensity-dependent data arrays measured for the different sequences. The time evolution of the system was simulated by numeric integration of the dynamic model with variable rate constants. Non-linear regressions were carried out simultaneously against all data points measured for the individual dimers. At this stage, our initial model failed (Fig. 3a, dashed curves): Neither the hyperbolic response of the steady state concentration nor the initial conversion rate changes upon the light intensity increase were captured correctly. Hyperbolic steady-state concentration dependence on light intensity can only be obtained for this reaction system by incorporating autocatalysis into the model ${ }^{27,28}$. Indeed, we cannot rule out the preferential foldamer segment interactions between the dimers and the monomers (Fig. 3b) for these low complexity and flexibly coupled peptides. This type of templating can exert autocatalysis on dimer formation by both proximity-controlled radical substitution (Fig. 3c, [12]) and concerted disulfide metathesis (Fig. 3c, [14]). The corresponding rate terms ( $v_{s, a l}$ and $v_{s, a 2}$ ) can be calculated (eqs. (6) and (7)). Details are provided in the Supplementary text.

$$
\begin{aligned}
& v_{s, a 1}=s_{a 1} I[\mathrm{MSSM}][\mathrm{MSSG}]^{2} \\
& v_{s, a 2}=s_{a 2} I^{2}[\mathrm{MSSM}][\mathrm{MSSG}]^{2}
\end{aligned}
$$

The autocatalytic radical substitution and concerted metathesis rate constants are $s_{a 1}$ and $s_{a 2}$, respectively. With eqs. (2)-(7), the steady-state concentration can be expressed in the refined model (eq. (8)).

$$
[\mathrm{MSSM}]_{s s}=\frac{\left(s_{p 1} \sqrt{I}+s_{p 2} I^{1.5}\right)[\mathrm{MSSG}]^{2}}{b-\left(s_{a 1} \sqrt{I}+s_{a 2} I^{1.5}\right)[\mathrm{MSSG}]^{2}}
$$

Thus, the light-intensity dependence also appears in the denominator explaining the hyperbolic function observed in the experiments. The hyperbolic approximation is valid only at low conversions $\left([\mathrm{MSSG}] \approx[\mathrm{MSSG}]_{0}\right.$ ), which holds for our experiments. Beyond the steady-state analysis, the dynamic model incorporating autocatalysis (eqs. (2)-(7)) was used to numerically simulate and fit the time- and light intensity-dependent data arrays. Excellent agreement was found this time (Fig. 3a, solid curves and Supplementary Fig. 2). Time evolution of the reaction rates revealed that the autocatalytic pathway dominates dimer synthesis after 10 min (Fig. 3d). The proximity-controlled, spontaneous (non-autocatalytic) mechanism is effective in seeding but itself would not produce detectable dimer formation within the observed time frame. Inspection of the resulting rate constants indicated that both the proximity-controlled radical substitution and the concerted metathesis mechanisms are present in the system. At the level of the individual dimers, however, the mechanism appeared to be dependent on the sequence (Supplementary Table 2). Understanding this substituent-dependent mechanism requires further investigation, which is beyond the scope of the present work.

These results strongly support the notion that the photochemical disulfide exchange carried out with the self-assembling foldamers drives autocatalytic synthesis, beyond the proximitycontrolled spontaneous coupling and breakdown pathways. 
Figure 3. Effects of templated autocatalysis on dimer formation. a) The best-fitting dynamic models with (solid) and without (dashed curves) autocatalysis. Representative experimental time- and light intensity-dependent data array is shown for the dimer WF-YF measured at power densities of $2.55 \mathrm{~mW} \mathrm{~cm}^{-2}$ (grey), $3.80 \mathrm{~mW} \mathrm{~cm}^{-2}$ (green) and $5.10 \mathrm{~mW} \mathrm{~cm}^{-}$ 2 (blue). b) Schematic representation of the templating dimer-monomer interaction. c) The proposed proximity-controlled autocatalytic reaction mechanism. (d) Time evolution of the reaction rates calculated for the proximity-controlled spontaneous synthesis $\left(v_{s, p}\right.$ : red) and the autocatalytic synthesis $\left(v_{s, a}\right.$ : blue) in the best-fitting dynamic model for WF-YF.

\section{Inverse-seeding experiments support the presence of templated cross-catalysis}

Templated auto-/cross-catalysis and spontaneous seeding are inseparable in this system due to the low complexity of the building blocks. Moreover, cross-catalysis may occur in the multicomponent reaction network with high probability. In principle, the total number of potential individual dimer - monomer pair combinations is 6084 . These conditions render the standard seeding experiments for testing autocatalysis unfeasible. To circumvent this combinatorial problem, we attempted to find monomer pairs for which synthesis relies predominantly on cross-catalysis. Isolation of such monomer pairs from the original mixture is expected to result in suppressed dimer production, time lags or both phenomena (hence inverse seeding). After sampling the dimer-producing population in the original experiment, we found that the monomer pairs WF-LW, WF-YF, RW-LW, KW-TW, YF-KW and RW-TW measured separately do not display dimer synthesis in $12 \mathrm{~h}$. When the corresponding six monomers were mixed (RW, LW, WF, YF, KW and TW), the dimers YF-KW and RW-TW could be detected at lower concentrations relative to the original population (Fig. 4). These observations confirm that templated cross-catalytic mechanism is present in the reaction 
network. The strongly sequence-dependent steady-state concentration of the dimers is in good agreement with the structural information transfer facilitated by the templated cross/autocatalysis.

The presence of the sequence-dependent templated cross-catalysis supports the conclusion that the foldamer disulfides behave as chemical replicators in the photochemical disulfide exchange reaction. However, the robust energy influx-dependent replication occurs only in a sufficiently large and diverse replicator population.

Figure 4. Dependence of dimer concentrations on the composition of the starting monomer population: inverse seeding. Time-dependent concentrations for dimers RW-TW (a) and YF-KW (b), starting from different monomer populations. Results obtained for the original population of twelve monomers (orange curve), the reduced population of six monomers (RW-SG, LW-SG, WF-SG, YF-SG, KW-SG and TW-SG) (blue curve) and the separate monomer pairs (black curve).

\section{Light intensity-dependent steady state replicator concentrations are off-equilibrium: dissipative system}

In this system, the kinetic asymmetry is dependent on the intensity of the energy influx; therefore, the replicator concentrations showed marked changes with the light intensity. This is a characteristic of a dissipative system. To gain further evidence for dissipative replication, we directly compared the dimer concentrations in the equilibrium attained through the reversible thiolate-mediated exchange with those obtained in the kinetically asymmetric photochemical rearrangement. To enable the visualisation of the significant differences, we calculated the logarithm of the concentration ratios $\left(\log \left(c_{\text {Dissip }} / c_{E q u i}\right)\right)$ for each dimer and 
displayed the values on a heat map (Fig. 5). At 75\% light intensity, the steady-state replicator concentrations are well below the equilibrium values (Fig. 5a), which is due to the strong bias toward the replicator decomposition mechanism. This finding shows that the replicator system is off-equilibrium already at low energy influx, but replicator breakdown is the predominant process. At 100\% light intensity, this situation fundamentally changes because light energydriven replication successfully competes with the decomposition mechanism (Fig. 5b). The deviation from the equilibrium concentrations is strongly dependent on sequence. Hydrophobic sequences are less favoured in the dissipative system, whereas replicators with polar or cationic side chains reach higher concentrations relative to the equilibrium system. Likely, stabilisation of the equilibrium structures and the transition states of replication require different interaction geometry. However, further investigation is necessary to explain this phenomenon. We conclude that both the overall abundance and the sequence-dependent concentration profile of the replicators deviate from the equilibrium values. This finding, together with the inherent energy-influx dependence, supports the dissipative nature of the system.

Figure 5. Light intensity- and sequence-dependent deviation of the replicator concentrations from the equilibrium values. The logarithm of the concentration ratios $\left(\log \left(c_{\text {Dissip }} / c_{\text {Equi }}\right)\right)$ displayed on a heat map. $c_{\text {Dissip }}$ stands for the steady-state concentrations measured in the kinetically asymmetric photochemical exchange at $75 \%$ light intensity (a) and $100 \%$ light intensity (b). CEqui indicates the concentrations obtained in the thiolate-mediated exchange reaction relaxed to equilibrium. The combinations of monomers in the specific dimers are displayed as two-letter codes on the horizontal and vertical axes. 


\section{Discussion}

We successfully coupled light-harvesting thiyl radical chemistry with the molecular recognition processes occurring in a foldameric network. Disulfide linkage has a long history and its nucleophilic exchange mechanism is currently popular in systems chemistry applications ${ }^{29,30}$. The photocatalytic rearrangement reaction of disulfides has also been known for decades. ${ }^{16}$ However, our work demonstrates the ability of the UV light-induced cleavagerecombination cycle of the disulfide bond to drive kinetically asymmetric processes. The root of asymmetry is the binding between peptidic segments that facilitates a fast intracomplex route for the thiyl-mediated exchange reactions under proximity-control (Fig. 6a). In parallel, the diffusion-controlled chain reaction in the background effectively couples disulfides with substituents that rapidly diffuse without preferential binding (Fig. 6b). The weak interactions can exert proximity-control because the high-energy radical intermediates rapidly relax to the dimer before entering the chain reaction. This finding suggests that the weak binding between primitive structures facilitates kinetic asymmetry if the dynamic covalent rearrangement is fast enough $^{4,31}$. Since the competing mechanisms are affected by the light intensity in different orders, the light not only switched processes on and off, but the energy influx also influenced the extent of kinetic asymmetry. UVA irradiation thereby drives the system to off-equilibrium steady states, ratcheting up the fittest sequences ${ }^{2,32}$. Thus, the system meets the criteria of dissipativity.

Figure 6. Schematic representation of the mechanisms for the dissipative replication system. (a) Seeding via foldamer assembly and subsequent proximity-controlled radical substitution or concerted metathesis. (b) Replicator breakdown through diffusion-controlled radical substitution. (c) Replication with templated cross-/autocatalysis facilitating the proximity-controlled radical substitution or concerted metathesis. 
The modular nature of the foldameric disulfides allowed templating interactions between the foldamer-disulfide dimers and the glutathione-protected monomers. This interaction extends the proximity-controlled mechanism to an autocatalytic process, thereby facilitating dissipative replication (Fig. 6c). The dissipative replication and the replicator death mechanism asymmetrically compete without physical separation of the pathways ${ }^{33}, 34$. In this system, the tendency toward assembly into structures of increased complexity is amplified by the dissipative seeded replication. In contrast, rapid diffusion - a property associated with low complexity - promotes decomposition. Thus, dissipative adaptation ${ }^{4,35}$ in the present system helps elucidate the chemical mechanisms of spontaneous emergence of complexity by selection. Pioneering experiments with dissipative self-assembling systems established the principles that govern chemical energy ${ }^{36-38}$ or light-driven ${ }^{39,40}$ trajectories to off-equilibrium states that generate spatial proximity-based order. Peptide-disulfides in the photochemical exchange reaction can be an additional valuable tool to study dissipative self-organization of simple building blocks.

Although the system presented here displays features essential for chemical evolution, prebiotic chemistry is beyond the scope of this study. However, the chemical availability of primitive Cys-containing peptides is supported by prebiotic Cys-catalysed amino acid and peptide synthesis ${ }^{41,42}$. These findings make thiyl radical-mediated dissipative replication of short, folding peptidic sequences an intriguing mechanism ${ }^{43}$, potentially illuminating energetic aspects of the transition from prebiotic chemical networks to biotic evolution.

\section{Methods}

UV-fuelled disulfide exchange reaction. Twelve different MSSG disulfides were dissolved in $20 \mathrm{mM}$ HEPES, $150 \mathrm{mM} \mathrm{NaCl}, 2 \mathrm{mM} \mathrm{CaCl}_{2}(\mathrm{pH}=7.0)$, with each disulfide at a final concentration of $10 \mu \mathrm{M}$. The reaction mixture was transferred into quartz cuvettes having a PTFE stopper and kept under an argon atmosphere during the experiment. Solutions were stirred at $150 \mathrm{RPM}$ and kept at a constant temperature of $303 \pm 1 \mathrm{~K}$ with an air-cooling system (modified Jasco Jetstream 2 Plus Column Thermostat). The temperature was monitored with a laser gun thermometer. Continuous illumination of the samples was carried out with a UVL28 EL Series UV lamp (Analytic Jena US, Upland, CA). The distance dependence of the power density and the emission spectrum of the lamp were determined prior to initiating the experiments (Supplementary Fig. 3), and the irradiation intensity was controlled by the distance between the lamp and the sample (Supplementary Fig. 4). $200 \mu \mathrm{L}$ samples were taken from the reaction mixture at each time point. Placing the sample in the dark freezed the reaction; no relaxation to equilibrium occurred. Nitric oxide blocked the photocatalytic reaction. To eliminate any slow non-photocatalytic disulfide exchange reaction in the mixture until the analysis, $100 \mu \mathrm{L}$ of $10 \%$ TFA in water was added. The product distribution of the samples was analysed using HPLC/ESI-MS measurements.

LC-MS measurements and MS data analysis. LC-MS analysis was performed with a Dionex UltiMate 3000 HPLC system interfaced with an LTQ ion trap mass spectrometer (Thermo Electron Corp., San Jose, CA, USA). Samples were injected onto an Aeris ${ }^{\mathrm{TM}}$ Widepore XBC18 $(250 \times 4.6 \mathrm{~mm}$, particle size: $3.6 \mu \mathrm{m}$, pore size $100 \AA)$ analytical HPLC column using 
gradient elution 5-80\% solution $\mathrm{B}$ during $25 \mathrm{~min}$ at $0.7 \mathrm{~mL} \mathrm{~min}^{-1}$ flow rate. Eluent composition was $0.1 \%$ formic acid in distilled water (Solution A) and $0.1 \%$ formic acid in acetonitrile (Solution B). The MS instrument was operated in the positive-ion mode using the equipped HESI-II source with the following parameters: capillary temperature: $350^{\circ} \mathrm{C}$; spray voltage: $3.0 \mathrm{kV}$; source heater temperature: $250^{\circ} \mathrm{C}$; sheath gas flow: $30 \mathrm{~mL} \mathrm{~min}^{-1}$; aux gas flow: $10 \mathrm{~mL}$ $\mathrm{min}^{-1}$. Mass spectra were acquired in full-scan mode from 200 to $2000 \mathrm{~m} / \mathrm{z}$. Thermo Xcalibur 2.2 software was used for peak identification and integration. $96 \%$ of the foldameric building blocks could be resolved independently by HPLC-MS/MS measurements based on molecular weight, MS fragmentation pattern and retention time depending on the relative hydrophobicity of the side chains. Those components which could not be resolved independently were integrated and averaged. A representative raw file for the library was utilised to create a processing method, where each sample component was associated with a chromatographic peak based on the previously identified mass $(\mathrm{m} / \mathrm{z}$ ) and retention time (Supplementary Table 1). Using the ICIS peak detection algorithm, the general detection and integration criteria were: smoothing points: 5; baseline window: 60; area noise factor: 5; peak noise factor: 10 . All raw data files were reprocessed with these processing setups together and analysed. Errors in peak identification during the automatic processing were corrected manually. 


\section{References}

1. Adamski, P. et al. From self-replication to replicator systems en route to de novo life. Nat. Rev. Chem. 4, 386-403 (2020).

2. Ragazzon, G. \& Prins, L.J. Energy consumption in chemical fuel-driven self-assembly. Nat. Nanotechnol. 13, 882-889 (2018).

3. Singh, N., Formon, G.J., De Piccoli, S. \& Hermans, T.M. Devising Synthetic Reaction Cycles for Dissipative Nonequilibrium Self-Assembly. Adv. Mater. 32, 1906834 (2020).

4. England, J.L. Dissipative adaptation in driven self-assembly. Nat. Nanotechnol. 10, 919-923 (2015).

5. Mattia, E. \& Otto, S. Supramolecular systems chemistry. Nat. Nanotechnol. 10, 111-119 (2015).

6. van Rossum, S.A.P., Tena-Solsona, M., van Esch, J.H., Eelkema, R. \& Boekhoven, J. Dissipative outof-equilibrium assembly of man-made supramolecular materials. Chem. Soc. Rev. 46, 5519-5535 (2017).

7. Rieß, B., Grötsch, R.K. \& Boekhoven, J. The design of dissipative molecular assemblies driven by chemical reaction cycles. Chem 6, 552-578 (2020).

8. $\quad$ Sorrenti, A., Leira-Iglesias, J., Sato, A. \& Hermans, T.M. Non-equilibrium steady states in supramolecular polymerization. Nat. Commun. 8, 15899 (2017).

9. Kathan, M. \& Hecht, S. Photoswitchable molecules as key ingredients to drive systems away from the global thermodynamic minimum. Chem. Soc. Rev. 46, 5536-5550 (2017).

10. Nanda, J. et al. Emergence of native peptide sequences in prebiotic replication networks. Nat. Commun. 8, 434 (2017).

11. Green, L.N. et al. Autonomous dynamic control of DNA nanostructure self-assembly. Nat. Chem. 11, 510-520 (2019).

12. Liu, Z. et al. Harnessing chemical energy for the activation and joining of prebiotic building blocks. Nat. Chem. 12, 1-6 (2020).

13. Morrow, S.M., Colomer, I. \& Fletcher, S.P. A chemically fuelled self-replicator. Nat. Commun. 10, 1011 (2019).

14. Monreal Santiago, G., Liu, K., Browne, W.R. \& Otto, S. Emergence of light-driven protometabolism on recruitment of a photocatalytic cofactor by a self-replicator. Nat. Chem. 12, 603-607 (2020).

15. Dadon, Z., Samiappan, M., Wagner, N. \& Ashkenasy, G. Chemical and light triggering of peptide networks under partial thermodynamic control. Chem. Commun. 48, 1419-1421 (2012).

16. Dinesh Gupta, A.R.K. Reactions of thiyl radicals. XIII. Photochemically induced exchange reactions of liquid alkyl disulfides. Can. J. Chem. 58, 1350-1354 (1980).

17. Li, J., Carnall, J.M.A., Stuart, M.C.A. \& Otto, S. Hydrogel Formation upon Photoinduced Covalent Capture of Macrocycle Stacks from Dynamic Combinatorial Libraries. Angew. Chem. Int. Edit. 50, 8384-8386 (2011).

18. Du, X. et al. Reversible and Rewritable Surface Functionalization and Patterning via Photodynamic Disulfide Exchange. Adv. Mater. 27, 4997-5001 (2015).

19. Klepel, F. \& Ravoo, B.J. Dynamic covalent chemistry in aqueous solution by photoinduced radical disulfide metathesis. Org. Biomol. Chem. 15, 3840-3842 (2017).

20. Lee, D.H., Granja, J.R., Martinez, J.A., Severin, K. \& Ghadiri, M.R. A self-replicating peptide. Nature 382, 525-528 (1996).

21. Amit, M., Yuran, S., Gazit, E., Reches, M. \& Ashkenasy, N. Tailor-Made Functional Peptide SelfAssembling Nanostructures. Adv. Mater. 30, 1707083 (2018).

22. Issac, R. \& Chmielewski, J. Approaching exponential growth with a self-replicating peptide. J. Am. Chem. Soc. 124, 6808-6809 (2002).

23. Tököli, A. et al. Proteomimetic surface fragments distinguish targets by function. Chem. Sci. 11, 10390-10398 (2020).

24. Fisher, B.F., Hong, S.H. \& Gellman, S.H. Helix Propensities of Amino Acid Residues via Thioester Exchange. J. Am. Chem. Soc. 139, 13292-13295 (2017).

25. Martinek, T.A. et al. Effects of the alternating backbone configuration on the secondary structure and self-assembly of beta-peptides. J. Am. Chem. Soc. 128, 13539-13544 (2006).

26. Bartus, É. et al. De Novo Modular Development of a Foldameric Protein-Protein Interaction Inhibitor for Separate Hot Spots: A Dynamic Covalent Assembly Approach. ChemistryOpen 6, 236-241 (2017).

27. Higgs, P.G. Chemical Evolution and the Evolutionary Definition of Life. J. Mol. Evol. 84, 225-235 (2017).

28. von Kiedrowski, G. Minimal Replicator Theory I: Parabolic Versus Exponential Growth, in Bioorganic Chemistry Frontiers. (eds. H. Dugas \& F.P. Schmidtchen) 113-146 (Springer Berlin Heidelberg, Berlin, Heidelberg; 1993). 
29. Diemer, V. et al. A cysteine selenosulfide redox switch for protein chemical synthesis. Nat. Commun. 11, 2558 (2020).

30. Canal-Martín, A. \& Pérez-Fernández, R. Biomimetic selenocystine based dynamic combinatorial chemistry for thiol-disulfide exchange. Nat. Commun. 12, 163 (2021).

31. Maiti, S., Fortunati, I., Ferrante, C., Scrimin, P. \& Prins, L.J. Dissipative self-assembly of vesicular nanoreactors. Nat. Chem. 8, 725-731 (2016).

32. Astumian, R.D. Kinetic asymmetry allows macromolecular catalysts to drive an information ratchet. Nat. Commun. 10, 1-14 (2019).

33. Corbett, A.D., Cheeseman, J.D., Kazlauskas, R.J. \& Gleason, J.L. Pseudodynamic Combinatorial Libraries: A Receptor-Assisted Approach for Drug Discovery. Angew. Chem. Int. Edit. 43, 2432-2436 (2004).

34. Ji, Q., Lirag, R.C. \& Miljanic, O.S. Kinetically controlled phenomena in dynamic combinatorial libraries. Chem. Soc. Rev. 43, 1873-1884 (2014).

35. Ashkenasy, G., Hermans, T.M., Otto, S. \& Taylor, A.F. Systems chemistry. Chem. Soc. Rev. 46, 25432554 (2017).

36. Liu, L. et al. Enzyme-free synthesis of natural phospholipids in water. Nat. Chem. 12, 1029-1034 (2020).

37. Pezzato, C. \& Prins, L.J. Transient signal generation in a self-assembled nanosystem fueled by ATP. Nat. Commun. 6, 7790 (2015).

38. Boekhoven, J., Hendriksen, W.E., Koper, G.J., Eelkema, R. \& van Esch, J.H. Transient assembly of active materials fueled by a chemical reaction. Science 349, 1075-1079 (2015).

39. Remon, P. et al. Light-driven control of the composition of a supramolecular network. Chem. Commun. 55, 4335-4338 (2019).

40. Kundu, P.K. et al. Light-controlled self-assembly of non-photoresponsive nanoparticles. Nat. Chem. 7 , 646-652 (2015).

41. Canavelli, P., Islam, S. \& Powner, M.W. Peptide ligation by chemoselective aminonitrile coupling in water. Nature 571, 546-549 (2019).

42. Foden, C.S. et al. Prebiotic synthesis of cysteine peptides that catalyze peptide ligation in neutral water. Science 370, 865-869 (2020).

43. Guseva, E., Zuckermann, R.N. \& Dill, K.A. Foldamer hypothesis for the growth and sequence differentiation of prebiotic polymers. Proc. Natl. Acad. Sci. USA 114, E7460 (2017).

\section{Data availability}

All data is available in the main text or the supplementary materials. All data and materials used in the analysis are available to any researcher for purposes of reproducing or extending the analysis.

\section{Acknowledgements}

Dr. Miklós Erdélyi (University of Szeged) is acknowledged for the calibration of the UV light source used for the measurements.

\section{Funding}

This research was funded by the National Research, Development and Innovation Office of Hungary, grant number GINOP-2.2.1-15-2016-00007 and NKFIA K134754. T.A.M. acknowledges support from the Hungarian Academy of Sciences LENDULET-Foldamer. Ministry of Human Capacities, Hungary grant 20391-3/2018/FEKUSTRAT is acknowledged.

\section{Author contributions}

B.M., Á.B. and É.B. synthesised the foldameric building blocks, performed the dissipative chemical network experiments and analysed the data. A.T. and E.W. analysed the foldamerfoldamer interactions, performed inverse seeding and evaluated the data. G.K. and Z.K. carried 
out foldamer stability test. G.F. contributed to the formulation of the concept and wrote the manuscript. T.A.M. developed the concept, designed the experiments, evaluated the experimental results and wrote the manuscript.

\section{Competing interests}

The authors declare no competing interests. 


\section{Supplementary Information for}

\section{Light-fuelled dissipative replication and selection in adaptive biomimetic chemical networks}

Éva Bartus ${ }^{1,2}$, Beáta Mag ${ }^{1}$, Áron Bajcsi ${ }^{1}$, Attila Tököli ${ }^{1}$, Gábor Kecskeméti ${ }^{1}$, Edit Wéber ${ }^{1}$, Zoltán Kele $^{1}$, Gabriel Fenteany ${ }^{1,3}$, Tamás A. Martinek ${ }^{1,2} *$

\footnotetext{
Affiliations:

${ }^{1}$ Department of Medical Chemistry, University of Szeged, Dóm tér 8, H-6720 Szeged, Hungary

${ }^{2}$ MTA-SZTE Biomimetic Systems Research Group, University of Szeged, Dóm tér 8, H-6720 Szeged, Hungary

${ }^{3}$ Institute of Genetics, Biological Research Centre, Temesvári krt. 62, H-6726 Szeged, Hungary

*Corresponding author. Email: martinek.tamas@med.u-szeged.hu
} 


\section{Table of Contents}

Supplementary Text ............................................................................................................. 3

Equation (1): Rate of spontaneous dimer synthesis through diffusion-controlled radical

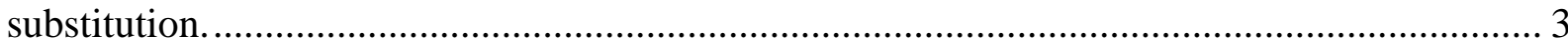

Equation (2): Rate of dimer breakdown through diffusion-controlled radical substitution........ 3 Equations (3) and (4): Rates of dimer synthesis through proximity-controlled mechanisms.... 4 Equations (5) and (6): Rates of autocatalytic dimer synthesis....................................... 5

Dynamic model for the foldamer-based photochemical disulphide exchange system .............. 6

Fitting the dynamic model to the experimental time- and energy-dependent data arrays .......... 6

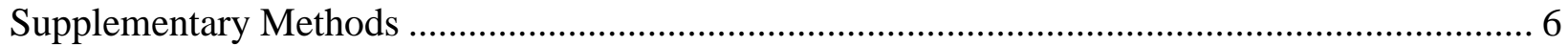

Synthesis and purification of the foldameric sequences ............................................. 6

Synthesis and purification of the glutathione protected monomers ...................................... 7

Generating statistical dimer distribution and calibration of the MS-AUC-to-concentration

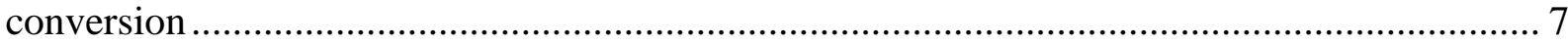

Generating equilibrium dimer distribution through thiolate-mediated exchange.................... 8

Calculation of the light intensity-dependent amplification factor ......................................... 8

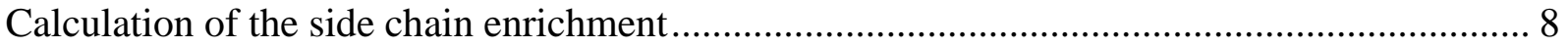

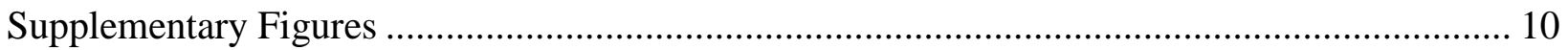

Supplementary Figure 1. Equilibrium product distribution of foldameric dimers in water. .... 10 Supplementary Figure 2. Fitting of the dynamic model to the light intensity and time-depent data arrays measured for representative replicators...................................................... 11

Supplementary Figure 3. Calibration of the UV light source. ......................................... 12

Supplementary Figure 4. Photo and schematic representation of the experimental setup....... 12 Supplementary Figure 5. Product distribution obtained by slow oxidizitation in chaotropic

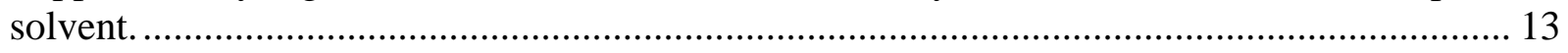

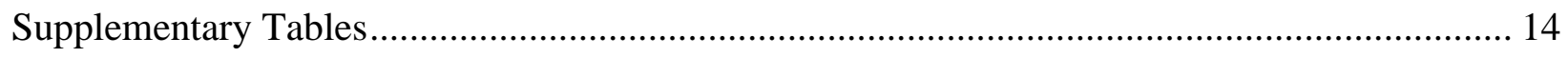

Supplementary Table 1. HPLC-MS characterisation of dimers (MSSM) and monomers

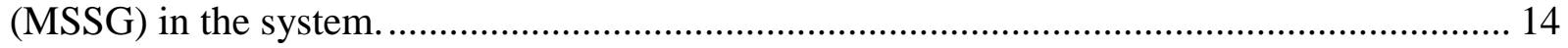

Supplementary Table 2. Fitted rate constants for representative dimers. ............................ 16

Supplementary Table 3. Characterisation of the glutathion-protected monomers. ................. 17

Supplementary Table 4. Calibration of the MS Area Under Curve (AUC) to concentration

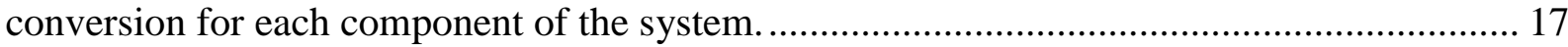

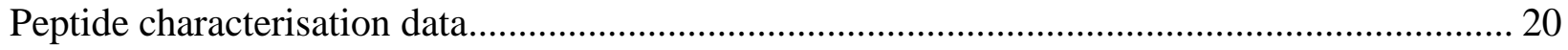

HPLC and MS characterization of the individual monomeric sequences ........................... 20

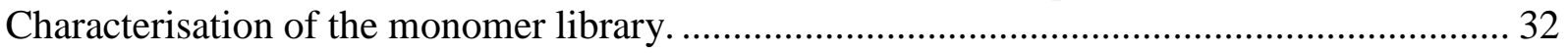

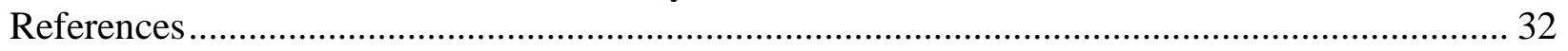




\section{Supplementary Text}

\section{Equation (1): Rate of spontaneous dimer synthesis through diffusion-controlled radical substitution.}

In this mechanism, dimers (MSSM) are produced by reaction of freely diffusing monomer radicals (MS·) with glutathione-protected monomers (MSSG) as shown in reaction [3a] (Fig. 1b). Here, "MS" corresponds to a single foldameric sequence in the reaction network.

$$
\text { MSSG + MS' } \longrightarrow \text { MSSM + GS• }
$$

The reaction rate for [3a] $\left(v_{s, c h}\right)$ satisfies eq. (S1),

$$
v_{s, c h}=k_{c h}[\mathrm{MS} \cdot][\mathrm{MSSG}]
$$

where $k_{c h}$ is the rate constant for the radical substitution step. In radical chain reactions, [MS·] can be approximated with a quasi-steady state approach. At low conversions, MS- is produced preferentially by homolytic cleavage of the starting material MSSG. Therefore, the rate of formation is proportional to the light intensity $\left(k_{I} I\right)$ and [MSSG]. The chain-termination steps consuming MS involve collisions with MS and GS. Again, the large excess of MSSG at low conversions affords the approximation that reaction [1a] (Fig. 1b) is dominant. Thus, there is a 12× molar excess for [GS·], that is, [GS·] $\approx 12[\mathrm{MS} \cdot$ ]. Consequently, the chain-termination rate can be expressed as a second order function in [MS·] (eq. (S2)).

$$
\begin{gathered}
\frac{d[\mathrm{MS} \cdot]}{d t}=0=k_{I} I[\mathrm{MSSG}]-k_{t}[\mathrm{MS} \cdot]^{2}-k_{t} 12[\mathrm{MS} \cdot]^{2} \\
{[\mathrm{MS} \cdot]=\sqrt{\frac{k_{I} I}{13 k_{t}}[\mathrm{MSSG}]}}
\end{gathered}
$$

Here, $k_{l}$ is the rate constant of the light-induced homolytic cleavage, and $k_{t}$ is the rate constant for the chain-termination reaction. Substituting eq. (S3) into eq. (S1) and collecting the concentrationand light intensity-independent term into the constant $s_{c h}$, the rate is given by eq. (1).

$$
v_{s, c h}=s_{c h} \sqrt{I}[\mathrm{MSSG}]^{1.5}
$$

\section{Equation (2): Rate of dimer breakdown through diffusion-controlled radical substitution.}

The decomposition of MSSM proceeds in this mechanism through the reaction between the freely diffusing glutathione radical (GS.) and MSSM (reaction [3b]).

$$
\mathrm{MSSM}+\mathrm{GS}^{\bullet} \longrightarrow \mathrm{MSSG}^{-\mathrm{MS}^{\bullet}}
$$

The rate equation can be expressed as follows (eq. (S4)). 


$$
v_{b}=k_{b}[\mathrm{GS} \cdot][\mathrm{MSSM}]
$$

For [GS·], we apply the quasi-steady state approximation again. The source of [GS·] is the lightinduced homolytic cleavage of MSSG and GSSG. The chain-termination steps consuming GS.

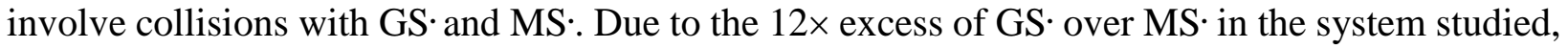
the latter reaction can be neglected. Thus, we obtain eq. (S5).

$$
\frac{d[\mathrm{GS} \cdot]}{d t}=0=k_{I} I([\mathrm{MSSG}]+2[\mathrm{GSSG}])-k_{t}[\mathrm{GS} \cdot]^{2}
$$

Due to the mass balance for the 'GS' moiety, the term [MSSG] + 2[GSSG] is constant and equals to the initial concentration of MSSG ([MSSG $]_{0}$ ), which yields eq. (S6).

$$
[\mathrm{GS} \cdot]=\sqrt{\frac{k_{I} I}{k_{t}}[\mathrm{MSSG}]_{0}}
$$

Thus, [GS·] is proportional to the square root of the light intensity, and eq. (S6) can be substituted into (S4). Collecting the constant term into the overall rate constant of $b$, we obtain eq. (2).

$$
v_{b}=b \sqrt{I}[\mathrm{MSSM}]
$$

\section{Equations (3) and (4): Rates of dimer synthesis through proximity-controlled mechanisms.}

This mechanism begins with the association preequilibrium producing the complex (MSSG)2.

$$
\begin{aligned}
2 \mathrm{MSSG} & \rightleftharpoons(\mathrm{MSSG})_{2} \\
{\left[(\mathrm{MSSG})_{2}\right] } & =\frac{1}{K_{D, p}}[\mathrm{MSSG}]^{2}
\end{aligned}
$$

$K_{D, p}$ stands for the dissociation constant. The rate-determining step is the photochemical cleavage of a monomer within the complex (reaction [6]). If the geometry of the complex is advantageous, the resulting high-energy radical can rapidly relax through the intracomplex radical substitution [7].

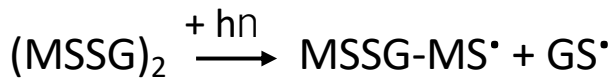

$$
\begin{aligned}
& \text { MSSG-MS } \longrightarrow \text { MSSM + GS }
\end{aligned}
$$

The rate of formation for MSSG-MS· is proportional to the light intensity $\left(k_{I} I\right)$ and [(MSSG) $)_{2}$. Using eq. (S7), the rate equation can be obtained (eq. (S8)).

$$
v_{s, p 1}=\frac{k_{I} I}{K_{D, p}}[\mathrm{MSSG}]^{2}
$$


Substituting the constant term with the overall rate constant $s_{p l}$ yields eq. (3).

$$
v_{s, p 1}=s_{p 1} I[\mathrm{MSSG}]^{2}
$$

Literature results showed that recombination of two thiyl radicals is a possible photochemical exchange mechanism, when radical substitution is sterically hindered ${ }^{1}$. Therefore, the intracomplex version of this mechanism cannot be ruled out a priori. If the absorption of the two photons is consecutive, the reaction rate remains first order in light-intensity; that is, the functional form of the rate equation is the same as eq. (3). If coincident absorption of two photons produces the diradical intermediate (MS $)_{2}$ and MSSM is formed in a concerted manner (steps [8] and [9]), the probability of the interaction with two photons is proportional to the square of the light intensity.

$$
\begin{gathered}
\left(\mathrm{MSSG}_{2} \stackrel{+2 \mathrm{~h}}{\longrightarrow}\left(\mathrm{MS}^{*}\right)_{2}+2 \mathrm{GS}^{\circ}\right. \\
\left(\mathrm{MS}^{*}\right)_{2} \stackrel{-}{\longrightarrow} \mathrm{MSSM}
\end{gathered}
$$

The cross-section of the interaction is not decreased by the non-linear "two-photon absorption" effect, because disulphides in the complex are not coupled quantum mechanically. Moreover, the recombination of the diradical [9] does not involve any activation energy, leading to a very fast reaction. On this ground, we incorporated this mechanism into the model with the rate equation (4).

$$
v_{s, p 2}=s_{p 2} I^{2}[\mathrm{MSSG}]^{2}
$$

\section{Equations (5) and (6): Rates of autocatalytic dimer synthesis.}

The autocatalytic mechanism starts with a binding preequilibrium between the dimers and the monomers ([10]). The dissociation constant $\left(K_{D, a}\right)$ determines the concentration of the complex available for the further, rate determining step.

$$
\begin{aligned}
2 \mathrm{MSSG}+\mathrm{MSSM} & \rightleftharpoons \mathrm{MSSM}-(\mathrm{MSSG})_{2} \\
{\left[\mathrm{MSSM}-(\mathrm{MSSG})_{2}\right] } & =\frac{1}{K_{D, a}}[\mathrm{MSSM}][\mathrm{MSSG}]^{2}
\end{aligned}
$$

The calculation of the rates for the autocatalytic routes is closely analogous to the non-autocatalytic synthesis pathways described above. For the intracomplex radical-substitution pathway, the ratedetermining step is the absorption of a photon, which leads to a linear light-intensity dependence of the reaction rate. Using (S9), eq. (6) is obtained.

$$
v_{s, a 1}=s_{a 1} I[\mathrm{MSSM}][\mathrm{MSSG}]^{2}
$$

Coincident absorption and the concerted conversion to MSSM is also possible for the autocatalytic complex, which yields a rate equation quadratic in light intensity (eq (7)).

$$
v_{s, a 2}=s_{a 2} I^{2}[\mathrm{MSSM}][\mathrm{MSSG}]^{2}
$$




\section{Dynamic model for the foldamer-based photochemical disulphide exchange system}

The mathematical framework of chemical evolution ${ }^{2,3}$ was invoked to analyse the experimentally determined energy-dependent dynamics of the dimer synthesis. The differential equation describing the time- and light intensity-dependent concentration of the dimers ([MSSM]) contained the following rate terms (eq. (S10)): non-autocatalytic (spontaneous) synthesis ( $v_{s, p l}$ and $\left.v_{s, p 2}\right)$, autocatalytic synthesis $\left(v_{s, a l}\right.$ and $\left.v_{s, a 2}\right)$ and breakdown $\left(v_{b}\right)$.

$$
\frac{d[\mathrm{MSSM}]}{d t}=v_{s, p 1}+v_{s, p 2}+v_{s, a 1}+v_{s, a 2}-v_{b}
$$

Fitting the dynamic model to the experimental time- and energy-dependent data arrays

Differential equation (S10) was numerically integrated using the Runge-Kutta (RK4) method to simulate the light intensity-dependent time evolution of a dimer. The synthesis rates are dependent on the actual monomer concentration, which was expressed with [MSSM] using the mass balance (S11).

$$
[\mathrm{MSSG}]=[\mathrm{MSSG}]_{0}-2[\mathrm{MSSM}]
$$

The numeric integrations were carried out with the parameters of $[\mathrm{MSSM}]_{0}=0,[\mathrm{MSSG}]_{0}=2 \times 10^{-}$

${ }^{5} \mathrm{M}$ and $\Delta \mathrm{t}=4.17 \times 10^{-2} \mathrm{~h}$. The light intensities were set to the calibrated values. The rate constants $s_{p 1}, s_{p 2}, s_{a l}, s_{a 2}$ and $b$ were determined by fitting (non-linear regression) the simulated curves against the time- and light intensity-dependent experimental data array.

\section{Supplementary Methods}

\section{Synthesis and purification of the foldameric sequences}

Foldameric sequences having L-Gly-L-Gly-L-Cys C-terminal segments were synthesised manually by standard solid-phase peptide synthesis with Fmoc/tBu chemistry. Rink Amide AM resin was used as solid support (capacity: $0.71 \mathrm{mmol} / \mathrm{g}$ ) and HATU (1-[bis(dimethylamino)methylene]- $1 \mathrm{H}$ 1,2,3-triazolo[4,5-b]pyridinium 3-oxid hexafluorophosphate) as coupling reagent in the presence of DIEA ( $N, N$-diisopropylethylamine). Amino acids and coupling reagents were used in excess of 3 equivalents and shaking was applied at room temperature for $3 \mathrm{~h}$. Deprotection was carried out in a DMF ( $N, N$-dimethylformamide) solution containing $2 \%$ DBU (1,8-diazabicycloundec-7-ene) and $2 \%$ piperidine. Cleavage was performed with TFA/ $\mathrm{H}_{2} \mathrm{O} / \mathrm{DTT}$ (DL-dithiothreitol)/TIS (triisopropylsilane) (90:5:2.5:2.5), which was followed by precipitation in ice-cold diethyl ether. The resin was washed with acetic acid and water, filtered, then lyophilised. Peptides were purified by RP-HPLC on a C18 column (Phenomenex Luna C18, $250 \times 10.00 \mathrm{~mm}$, particle size: $10 \mu \mathrm{m}$, pore size: $100 \AA$ ). The HPLC eluents were $0.1 \%$ TFA in water (Eluent A), and $0.1 \%$ TFA/ 80\% ACN (acetonitrile) in water (Eluent B). Different gradient elution was used according to the 
hydrophobicity of the peptides. Purity was confirmed by analytical RP-HPLC and ESI-MS measurements.

\section{Synthesis and purification of the glutathione protected monomers}

Glutathione-protected monomers were synthesised by oxidative coupling of thiols in the solution phase. Each purified foldamer was dissolved in 20\% DMSO (dimethyl sulfoxide) in water separately to $1 \mathrm{mM}$ concentration in the presence of $20 \times$ excess of GSH (reduced glutathione) and stirred overnight at room temperature exposed to atmospheric oxygen. The completeness of the oxidation reaction was monitored by HPLC-MS, and the reaction mixture was injected directly onto a semi-preparative HPLC column (Phenomenex Luna C18, $250 \times 10.00 \mathrm{~mm}$; particle size: 10 $\mu \mathrm{m}$; pore size: $100 \AA$ ) and purified. Under this reaction condition the amount of the homodimeric foldamer was negligible and could be completely separated from the foldamer-glutathione adduct.

\section{LC-MS analysis of the purified glutathione protected monomers}

UHPLC-MS/MS measurements were used to characterize the pure peptides by using an ACQUITY I-Class UPLC ${ }^{\text {TM }}$ liquid chromatography system (Waters, Manchester, UK) coupled with a Q Exactive ${ }^{\mathrm{TM}}$ Plus Hybrid Quadrupole-Orbitrap Mass Spectrometer (Thermo Fisher Scientific, San Jose, CA, USA). Chromatographic separation was carried out at $25^{\circ} \mathrm{C}$ using $0.1 \%$ formic acid in water as solvent $\mathrm{A}$ and $\mathrm{ACN}$ containing $0.1 \%$ formic acid as solvent $\mathrm{B}$. The following multistep gradient was used: $5-50 \%$ over 20 minutes then $50-80 \%$ over 5 minutes and finally $80 \%$ solvent B for additional 5 minutes at $0.7 \mathrm{~mL} \mathrm{~min}^{-1}$ flow rate. Samples where incubated at $5^{\circ} \mathrm{C}$ until the measurement and $15 \mu \mathrm{L}$ of the sample was injected into the UHPLC-MS/MS system. The MS instrument was operated in the positive-ion mode using the equipped HESI-II source with the following parameters: capillary temperature: $256^{\circ} \mathrm{C}$; spray voltage: $3.5 \mathrm{kV}$; aux gas heater temperature: $412^{\circ} \mathrm{C}$; sheath gas flow: $47.5 \mathrm{~mL} \mathrm{~min}^{-1}$; aux gas flow: $11 \mathrm{~mL} \mathrm{~min}^{-1}$; and S-lens RF level, 50.0 (source auto-defaults). Full scan was conducted with a mass range of 150$2000 \mathrm{~m} / z$ with resolution of 70,000. The ACG (automatic gain control) setting was defined as $3 \times 10^{6}$ charges, and the maximum injection time was set to $100 \mathrm{~ms}$. Data dependent MS/MS was acquired in a mass range of $200-2000 \mathrm{~m} / \mathrm{z}$ with resolution of 17,500 . AGC setting was defined as $5 \times 10^{5}$ charges, and the maximum injection time was set to $150 \mathrm{~ms}$.

\section{Generating statistical dimer distribution and calibration of the MS-AUC-to-concentration conversion}

Statistical product distribution for the dimers were generated in a chaotropic solvent mixture of 20\% DMSO:80\% water. Glutathione-protected monomers were dissolved (each one at $10 \mu \mathrm{M}$ final concentration) and the library was completely reduced with 2 molar equivalents of TCEP (tris(2carboxyethyl)phosphine). Subsequently, the sample was continuously stirred for $48 \mathrm{~h}$ exposed to atmospheric oxygen.

To confirm that oxidation in the chaotropic solvent (DMSO:water) results in statistical product distribution, a subset of monomers was analysed for which self-association tendency is significantly different (Supplementary Fig. 1) (WF-SG, LW-SG, RW-SG and TW-SG). The oxidised mixture was analysed using HPLC-UV with the detection wavelength of $210 \mathrm{~nm}$, where 
the integrated intensity is directly proportional to the peptide concentration. For this system, the oxidation products could be quantitatively analysed in HPLC-UV without problem. As expected, the reaction yielded four homodimers and six heterodimers with statistical concentration distribution (Supplementary Fig. 5).

Next, we performed the same experiment starting from the 12-membered glutathione-protected foldamer disulphide library. Completeness of the oxidation after the TCEP treatment was confirmed, and the final product distribution was quantitatively analysed with HPLC-MS. Due to the large number of components, UV detection could not separate the products. Based on the model experiment, the statistical concentration of each dimer was predicted and then calculated. After the quantitative evaluation of the HPLC-MS chromatograms, the MS-AUC/concentration ratios were calculated for each component (Supplementary Table 4).

\section{Generating equilibrium dimer distribution through thiolate-mediated exchange}

The twelve different thiol-functionalized foldameric building blocks (IF-SH, KW-SH, LW-SH, QW-SH, RW-SH, RF-SH, SW-SH, TW-SH, VW-SH, WF-SH, WW-SH and YF-SH) were dissolved at a concentration of $10 \mu \mathrm{M}$ in a redox buffer (pH 8.0, $20 \mathrm{mM}$ HEPES, $150 \mathrm{mM} \mathrm{NaCl}$, $1 \mathrm{mM} \mathrm{CaCl}_{2}, 3 \mathrm{mM} \mathrm{NaN}_{3}, 500 \mu \mathrm{M} \mathrm{GSH}$ and $125 \mu \mathrm{M} \mathrm{GSSG}$ ). Reaction mixture was shaken at 250 $\mathrm{rpm}, 37^{\circ} \mathrm{C}$ for three days in a properly closed vial. Samples $(100 \mu \mathrm{L})$ were taken from the mixture every $24 \mathrm{~h}$, quenched with $10 \%$ TFA in water $(50 \mu \mathrm{L})$ and analysed with HPLC-MS ${ }^{4}$. Product distribution was obtained via quantitative evaluation of the HPLC-MS chromatograms and conversion of AUC-to-concentration was carried out as described above.

\section{Calculation of the light intensity-dependent amplification factor}

Light intensity-dependent amplification factors (AF) were calculated for each dimer to determine the sensitivity to the energy influx. The following formula (eq. (S12)) was used:

$$
A F_{i(C), i n t}=\frac{A U C_{i(C), 100 \%}}{A U C_{i(C), 50 \%}}
$$

where $A U C_{i(C), 100 \%}$ is the area under the curve (AUC) of compound $i$ measured at $100 \%$ light intensity $\left(5.10 \mathrm{~mW} \mathrm{~cm}{ }^{-2}\right)$ and $A U C_{i(C), 50 \%}$ is the AUC of the same compound measured at $50 \%$ light intensity $\left(2.55 \mathrm{~mW} \mathrm{~cm}^{-2}\right)$. Errors of $\mathrm{AF}_{i}\left(\sigma_{\mathrm{AF}}\right)$ were calculated from three parallel measurements with the following general formula (neglecting the correlation between the variables):

$$
\sigma_{A F i}=A F_{i} \sqrt{\left(\frac{\sigma_{A U C 1}}{A U C_{1}}\right)^{2}+\left(\frac{\sigma_{A U C 2}}{A U C_{2}}\right)^{2}}
$$

where $A U C_{1} \pm \sigma_{\mathrm{AUC} 1}$ and $A U C_{2} \pm \sigma_{\mathrm{AUC} 2}$ are the measured variables with uncertainties when $A F_{i}$ is calculated by $A U C_{1} / A U C_{2}$.

\section{Calculation of the side chain enrichment}


Enrichment $(\mathrm{E})$ of the proteinogenic side chains in the peripheral $\left(1,1^{\prime}\right)$ and the central $\left(2,2^{\prime}\right)$ positions of the ten most amplified dimers was expressed as the frequency of a side chain relative to all side chains in the foldamer system by using the following formula (S14):

$$
E_{\text {schi }}=\frac{N_{\text {schi,Best }} / 10}{N_{\text {schi,All }} / 78}
$$

Where $\mathrm{E}_{\text {schi }}$ is the enrichment of the side chain $i$ in appropriate position, $N_{\text {schiB Best }}$ is the count of the side chain $i$ among the top ten amplified dimers, $N_{\text {schi,All }}$ is the count of the side chain $i$ in the complete system. 


\section{Supplementary Figures}

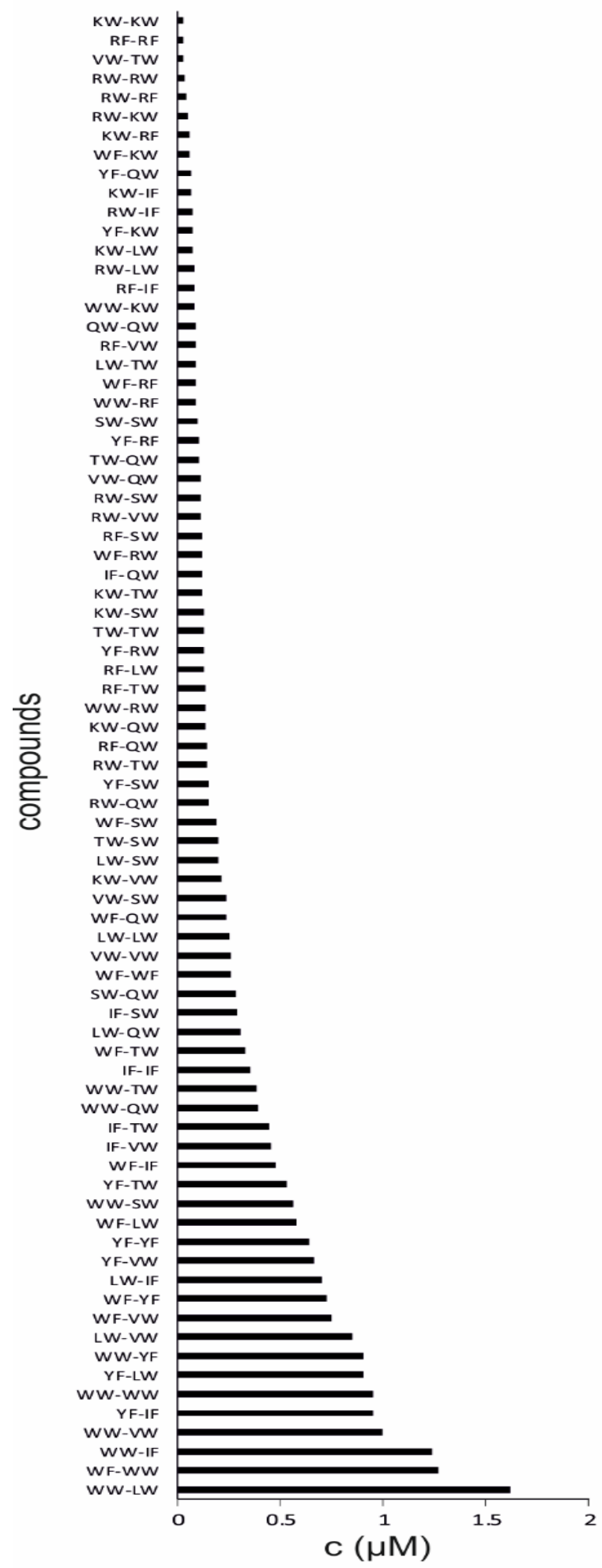

Supplementary Figure 1. Equilibrium product distribution of foldameric dimers in water. For the experimental conditions, see Supplementary Methods. 

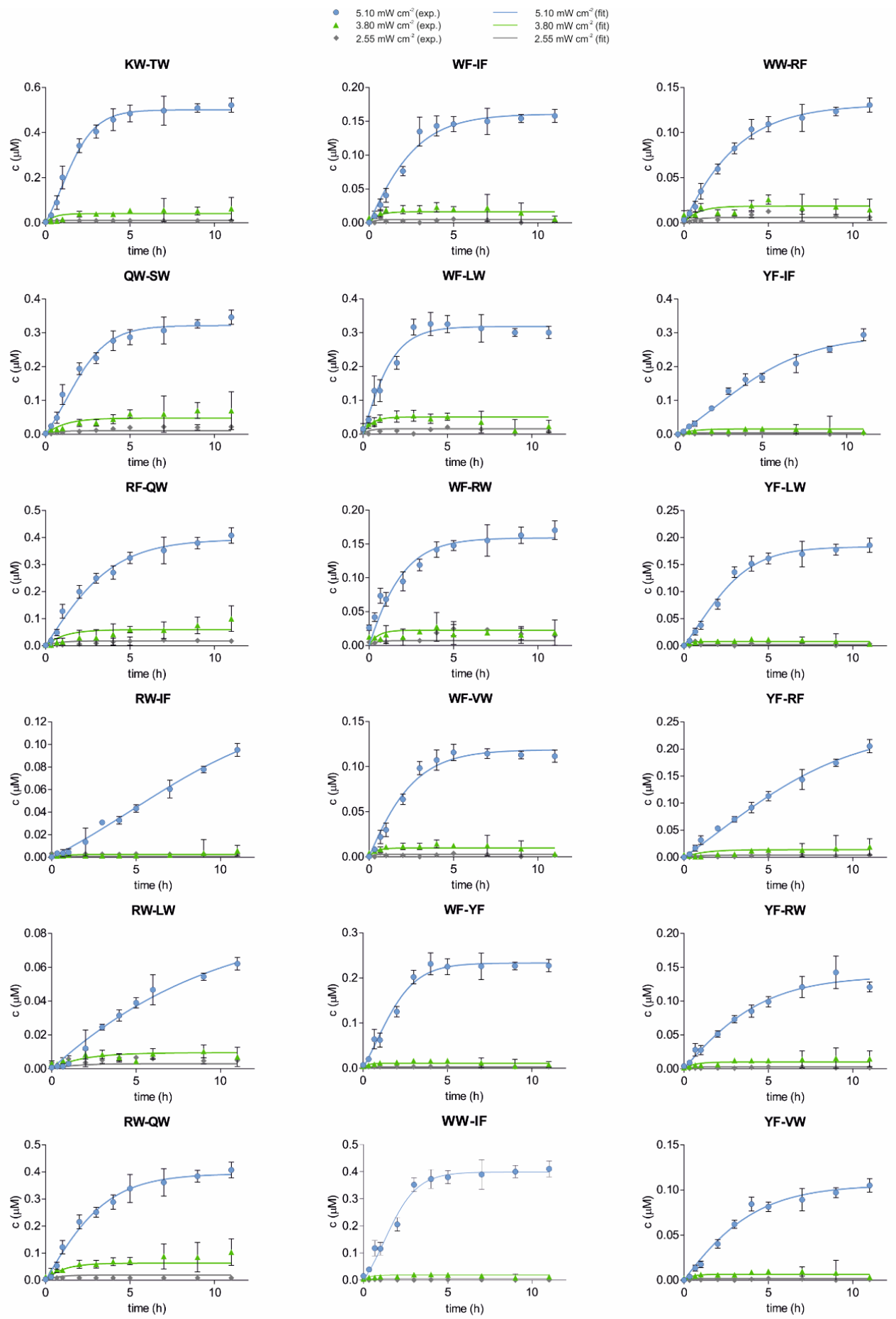

Supplementary Figure 2. Fitting of the dynamic model to the light intensity and time-depent data arrays measured for representative replicators. Data at different light intensities are represented as follows: $2.55 \mathrm{~mW} \mathrm{~cm}^{-2}$ (gray diamond), $3.80 \mathrm{~mW} \mathrm{~cm}^{-2}$ (green triangle) and 5.10 $\mathrm{mW} \mathrm{cm} \mathrm{cm}^{-2}$ (blue circle); fitted curves are depicted with matching color. Non-linear least-square analysis was used for fitting. Experimental data were obtained from three parallel measurements carried out on three different samples. See Supplementary Table 2 for fitted parameters. 

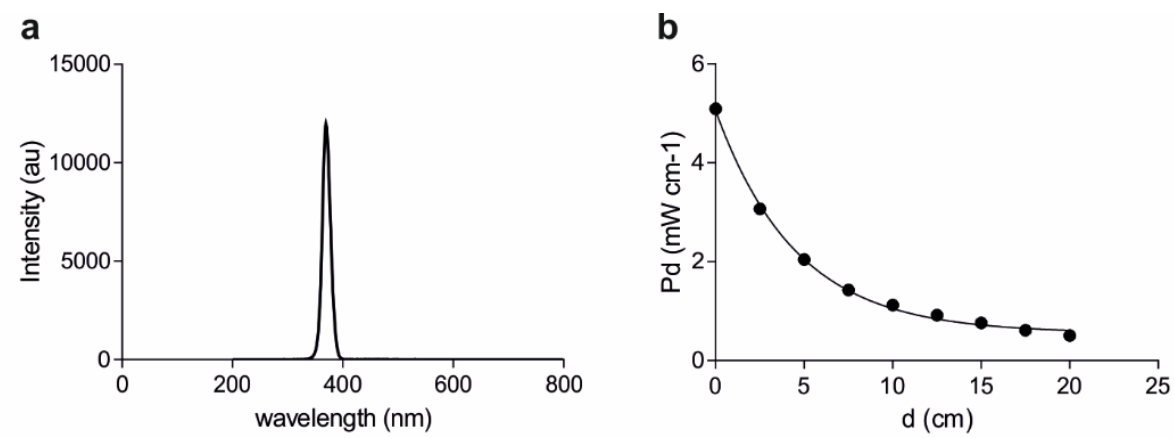

Supplementary Figure 3. Calibration of the UV light source. (a) Emission spectrum of the UV light source (UVL-28 EL Series UV Lamp), with an emission maximum at $365 \pm 5 \mathrm{~nm}$ and (b) dependence of the power density $\left(\mathrm{Pd}\left[\mathrm{mW} \mathrm{cm}^{-2}\right]\right)$ on the distance. Power density was measured with an S140C Integrating Sphere Photodiode Power Sensor (Thorlab Inc.). The wavelength range of the Si detector was 350-1100 nm, and the power range was $1 \mu \mathrm{W}-500 \mathrm{~mW}$ (resolution: $1 \mathrm{nW}$ )
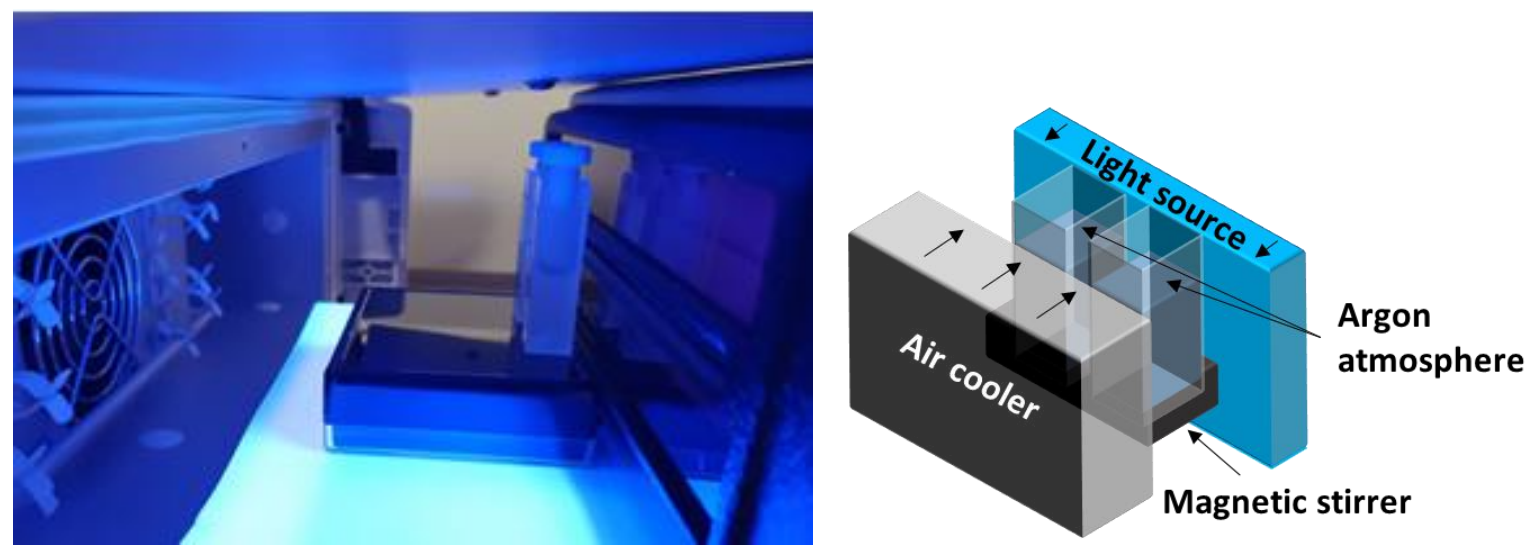

Supplementary Figure 4. Photo and schematic representation of the experimental setup. The reaction mixtures were stirred at 150 RPM in quartz cuvettes having a PTFE stopper and kept under argon atmosphere during the experiment. Constant temperature $(303 \pm 1 \mathrm{~K})$ was maintained with active air-cooling system (column thermostat). Temperature was monitored with laser-gun thermometer. Continuous irradiation of the samples was carried out by UVL-28 EL Series UV lamp working at $365 \mathrm{~nm}$ (Analytic Jena US, Upland, CA) and the power density was varied by changing the distance between the light source and the samples. 


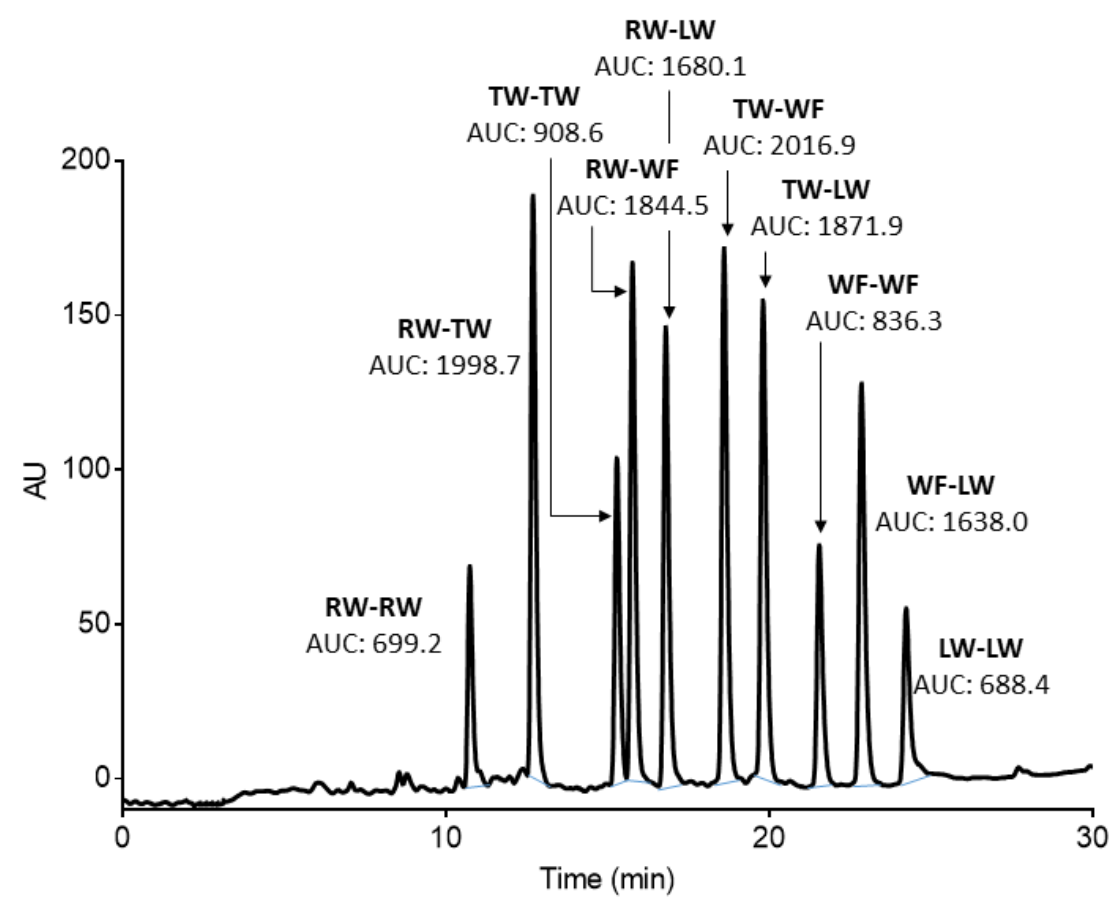

Supplementary Figure 5. Product distribution obtained by slow oxidization in chaotropic solvent.

Oxidation of the glutathione-protected foldamers WF-SG, LW-SG, RW-SG and TW-SG resulted in a statistical product distribution despite their different tendencies to associate. Samples were analysed by HPLC-MS. Conditions of the analytical HPLC-MS measurement: column: Phenomenex Luna-C18 $(250 \times 4.6 \mathrm{~mm})$; method: $5-80 \%$ B during $25 \mathrm{~min}$, flow rate: $0.7 \mathrm{~mL} \mathrm{~min}^{-}$ , where eluent A: 0.1\% TFA in water, eluent B: 0.1\% TFA in $\mathrm{ACN}$ : water = 8:1. 


\section{Supplementary Tables}

Supplementary Table 1. HPLC-MS characterisation of dimers (MSSM) and monomers (MSSG) in the system.

\begin{tabular}{|c|c|c|c|c|}
\hline & & \\
Compounds & $\begin{array}{c}\text { Calculated } \\
\text { molar } \\
\text { mass (Da) }\end{array}$ & $\begin{array}{c}\text { Retention } \\
\text { time } \\
(\mathrm{min})\end{array}$ & \multicolumn{2}{|c|}{ Detected ions } \\
\cline { 4 - 5 } & {$\left[\mathrm{M}+2 \mathrm{H}^{+}\right]^{2+}$} & {$\left[\mathrm{M}+3 \mathrm{H}^{+}\right]^{3+}$} \\
\hline IF-IF & 2044.66 & 17.73 & 1022.95 & 682.29 \\
\hline IF-QW & 2098.67 & 15.70 & 1049.79 & 700.47 \\
\hline IF-SG & 1327.62 & 14.49 & 664.84 & 443.54 \\
\hline IF-SW & 2057.62 & 15.79 & 1029.89 & 686.75 \\
\hline IF-TW & 2071.65 & 16.05 & 1036.42 & 691.59 \\
\hline IF-VW & 2069.67 & 17.29 & 1035.41 & 690.82 \\
\hline KW-IF & 2098.71 & 14.55 & 1049.79 & 700.00 \\
\hline KW-KW & 2152.76 & 11.70 & 1077.40 & 718.48 \\
\hline KW-LW & 2137.75 & 14.70 & 1069.44 & 713.56 \\
\hline KW-QW & 2152.72 & 12.73 & 1076.93 & 718.90 \\
\hline KW-RF & 2141.74 & 11.63 & 1071.41 & 714.82 \\
\hline KW-SG & 1381.69 & 10.42 & 691.85 & 461.56 \\
\hline KW-SW & 2111.63 & 12.73 & 1056.87 & 705.04 \\
\hline KW-TW & 2125.70 & 13.09 & 1063.35 & 709.34 \\
\hline KW-VW & 2123.68 & 14.26 & 1063.34 & 708.73 \\
\hline LW-IF & 2084.61 & 17.84 & 1042.52 & 695.41 \\
\hline LW-LW & 2123.72 & 18.00 & 1062.03 & 708.28 \\
\hline LW-QW & 2137.71 & 15.84 & 1069.41 & 713.77 \\
\hline LW-SG & 1366.68 & 14.76 & 684.35 & 456.56 \\
\hline LW-SW & 2096.66 & 15.94 & 1048.90 & 700.19 \\
\hline LW-TW & 2111.67 & 16.19 & 1056.01 & 704.69 \\
\hline LW-VW & 2108.71 & 17.43 & 1054.60 & 703.40 \\
\hline QW-QW & 2152.68 & 13.71 & 1077.12 & 718.79 \\
\hline QW-SG & 1381.65 & 11.60 & 691.83 & 461.55 \\
\hline RF-IF & 2087.69 & 14.59 & 1044.38 & 697.05 \\
\hline RF-LW & 2126.73 & 14.77 & 1063.94 & 709.58 \\
\hline RF-QW & 2141.70 & 12.73 & 1071.37 & 715.15 \\
\hline RF-RF & 2130.72 & 11.64 & 1066.67 & 710.97 \\
\hline RF-SG & 1370.68 & 10.31 & 686.35 & 457.89 \\
\hline RF-SW & 2100.65 & 12.80 & 1050.89 & 701.43 \\
\hline RF-TW & 2114.68 & 12.77 & 1057.93 & 706.26 \\
\hline RF-VW & 2112.70 & 14.28 & 1056.99 & 705.03 \\
\hline RW-IF & 2126.73 & 14.77 & 1064.57 & 709.87 \\
\hline RW-KW & 2180.78 & 11.80 & 1091.33 & 728.04 \\
\hline RW-LW & 2165.77 & 14.91 & 1083.50 & 722.76 \\
\hline RW-QW & 2180.74 & 12.90 & 1090.87 & 728.20 \\
\hline & 2169.76 & 12.22 & 1085.36 & 724.15 \\
\hline & & & & \\
\hline
\end{tabular}




\begin{tabular}{|c|c|c|c|c|}
\hline RW-RW & 2208.80 & 11.97 & 1104.90 & 737.47 \\
\hline RW-SG & 1409.69 & 10.56 & 705.85 & 470.90 \\
\hline RW-SW & 2139.69 & 12.96 & 1070.38 & 714.39 \\
\hline RW-TW & 2153.72 & 13.29 & 1077.46 & 718.90 \\
\hline RW-VW & 2151.74 & 14.41 & 1076.35 & 718.45 \\
\hline SW-QW & 2110.69 & 13.76 & 1056.41 & 704.63 \\
\hline SW-SG & 1340.63 & 11.60 & 671.32 & 447.88 \\
\hline SW-SW & 2070.58 & 12.99 & 1035.86 & 690.84 \\
\hline TW-QW & 2125.66 & 14.05 & 1063.25 & 709.33 \\
\hline TW-SG & 1354.64 & 12.06 & 678.33 & 452.55 \\
\hline TW-SW & 2083.70 & 14.13 & 1042.93 & 695.92 \\
\hline TW-TW & 2098.64 & 14.35 & 1049.99 & 700.56 \\
\hline VW-QW & 2122.74 & 15.35 & 1063.00 & 708.79 \\
\hline VW-SG & 1352.66 & 14.05 & 677.34 & 451.89 \\
\hline VW-SW & 2082.63 & 15.44 & 1042.40 & 695.14 \\
\hline VW-TW & 2096.66 & 15.70 & 1049.37 & 699.79 \\
\hline VW-VW & 2094.68 & 16.97 & 1048.03 & 698.88 \\
\hline WF-IF & 2117.72 & 17.27 & 1059.49 & 706.71 \\
\hline WF-KW & 2171.77 & 14.31 & 1086.48 & 724.64 \\
\hline WF-LW & 2156.76 & 17.45 & 1078.90 & 719.64 \\
\hline WF-QW & 2171.73 & 15.40 & 1086.93 & 724.91 \\
\hline WF-RF & 2160.75 & 14.37 & 1081.34 & 721.03 \\
\hline WF-RW & 2199.79 & 14.48 & 1100.43 & 734.51 \\
\hline WF-SG & 1400.66 & 14.29 & 701.34 & 467.89 \\
\hline WF-SW & 2130.68 & 15.49 & 1065.95 & 711.30 \\
\hline WF-TW & 2144.71 & 15.76 & 1073.20 & 715.93 \\
\hline WF-VW & 2142.73 & 16.96 & 1071.97 & 715.50 \\
\hline WF-WF & 2190.78 & 16.96 & 1095.93 & 731.76 \\
\hline WF-WW & 2229.81 & 16.41 & 1115.44 & 744.44 \\
\hline WF-YF & 2167.74 & 16.10 & 1084.52 & 723.76 \\
\hline WW-IF & 2156.75 & 16.78 & 1078.99 & 719.84 \\
\hline WW-KW & 2210.80 & 13.73 & 1105.93 & 738.15 \\
\hline WW-LW & 2195.79 & 16.89 & 1098.46 & 732.71 \\
\hline WW-QW & 2210.76 & 14.71 & 1105.98 & 737.87 \\
\hline WW-RF & 2199.78 & 14.48 & 1100.43 & 734.51 \\
\hline WW-RW & 2238.82 & 13.94 & 1120.36 & 747.47 \\
\hline WW-SG & 1439.67 & 12.86 & 720.84 & 480.89 \\
\hline WW-SW & 2169.71 & 16.08 & 1086.53 & 725.38 \\
\hline WW-TW & 2183.74 & 15.09 & 1092.55 & 728.13 \\
\hline WW-VW & 2181.76 & 16.39 & 1091.41 & 728.06 \\
\hline WW-WW & 2268.84 & 15.81 & 1135.45 & 757.47 \\
\hline WW-YF & 2206.77 & 15.53 & 1103.89 & 736.72 \\
\hline YF-IF & 2094.68 & 16.41 & 1047.94 & 699.11 \\
\hline YF-KW & 2148.73 & 13.54 & 1075.00 & 717.42 \\
\hline YF-LW & 2133.72 & 16.57 & 1067.86 & 712.64 \\
\hline
\end{tabular}




\begin{tabular}{|c|c|c|c|c|}
\hline YF-QW & 2148.69 & 14.50 & 1074.93 & 717.02 \\
\hline YF-RF & 2137.71 & 13.54 & 1069.47 & 713.51 \\
\hline YF-RW & 2176.75 & 13.71 & 1088.93 & 726.82 \\
\hline YF-SG & 1377.66 & 12.98 & 689.83 & 460.22 \\
\hline YF-SW & 2107.64 & 14.58 & 1054.34 & 703.55 \\
\hline YF-TW & 2121.67 & 14.84 & 1061.45 & 707.98 \\
\hline YF-VW & 2119.69 & 16.06 & 1060.47 & 707.47 \\
\hline YF-YF & 2144.70 & 15.22 & 1073.30 & 715.89 \\
\hline
\end{tabular}

*Analytical HPLC-MS measurement. Column: Aeris Widepore XB-C18 $(250 \times 4.6 \mathrm{~mm})$. Method: 5-80\% B during $25 \mathrm{~min}$, flow rate: $0.7 \mathrm{~mL} \mathrm{~min}^{-1}$, where eluent $\mathrm{A}: 0.1 \% \mathrm{HCOOH}$ in water, eluent B: $0.1 \% \mathrm{HCOOH}$ in acetonitrile.

\section{Supplementary Table 2. Fitted rate constants for representative dimers.}

\begin{tabular}{|c|c|c|c|c|c|c|}
\hline Compounds & $\begin{array}{c}\mathrm{S}_{\mathrm{p} 1} \\
\mathrm{M}^{-1}\left(\mathrm{~cm}^{2}\right) \\
\mathrm{W}^{-1} \mathrm{~s}^{-1}\end{array}$ & $\begin{array}{c}\mathrm{Sp}_{\mathrm{p} 2} \\
\mathrm{X} 10^{2} \mathrm{M}^{-1} \\
\left(\mathrm{~cm}^{2}\right)^{2} \mathrm{~W}^{-2} \mathrm{~s}^{-1}\end{array}$ & $\begin{array}{c}\mathrm{S}_{\mathrm{a} 1} \\
\mathrm{x} 10^{7} \mathrm{M}^{-2} \\
\left(\mathrm{~cm}^{2}\right) \mathrm{W}^{-1} \mathrm{~s}^{-1}\end{array}$ & $\begin{array}{c}\mathrm{S}_{\mathrm{a} 2} \\
\mathrm{x} 10^{10} \mathrm{M}^{-2} \\
\left(\mathrm{~cm}^{2}\right)^{2} \mathrm{~W}^{-2} \mathrm{~s}^{-1}\end{array}$ & $\begin{array}{c}\mathrm{b} \\
\mathrm{x} 10^{-2} \\
\left(\mathrm{~cm}^{2}\right)^{0.5} \\
\mathrm{~W}^{-0.5} \mathrm{~s}^{-1}\end{array}$ & $\begin{array}{c}\text { RMSD } \\
\times 10^{-9} \mathrm{M} \\
\mathrm{s}^{-1}\end{array}$ \\
\hline KW-TW & 0.00 & 38.03 & 0.01 & 205.16 & 2.80 & 19.97 \\
\hline QW-SW & 0.00 & 25.94 & 0.00 & 107.88 & 1.57 & 15.49 \\
\hline RF-QW & 0.00 & 25.80 & 0.00 & 77.01 & 1.12 & 24.99 \\
\hline RW-IF & 0.00 & 1.99 & 0.00 & 117.49 & 1.68 & 3.60 \\
\hline RW-LW & 1.27 & 0.00 & 0.00 & 70.29 & 1.05 & 4.50 \\
\hline RW-QW & 0.00 & 27.53 & 18.24 & 66.25 & 1.47 & 18.47 \\
\hline WF-IF & 5.43 & 0.00 & 247.59 & 0.00 & 6.95 & 10.53 \\
\hline WF-LW & 0.00 & 38.80 & 100.06 & 73.31 & 3.85 & 22.19 \\
\hline WF-RW & 2.73 & 14.65 & 0.00 & 143.86 & 2.21 & 17.27 \\
\hline WF-VW & 4.39 & 0.24 & 304.75 & 0.00 & 8.61 & 6.70 \\
\hline WF-YF & 0.00 & 13.41 & 270.42 & 0.00 & 7.45 & 11.26 \\
\hline WW-IF & 0.00 & 24.06 & 0.00 & 280.08 & 3.84 & 20.34 \\
\hline WW-RF & 4.38 & 0.00 & 155.16 & 0.57 & 4.42 & 15.14 \\
\hline YF-IF & 0.00 & 8.91 & 0.02 & 112.28 & 1.58 & 11.85 \\
\hline YF-LW & 4.01 & 0.04 & 270.87 & 0.00 & 7.53 & 7.15 \\
\hline YF-RF & 0.00 & 5.91 & 0.00 & 83.24 & 1.19 & 6.35 \\
\hline YF-RW & 2.83 & 0.35 & 177.91 & 0.08 & 5.01 & 6.71 \\
\hline YF-VW & 0.00 & 6.77 & 0.00 & 178.50 & 2.64 & 5.06 \\
\hline
\end{tabular}

Mean standard deviation of the fitted parameters was estimated with jackknife resampling technique and it was found to be less than $3.4 \%$ in each case.

$\mathrm{s}_{\mathrm{p} 1}$ : rate constant of spontaneous synthesis by proximity-controlled radical substitution $\mathrm{s}_{\mathrm{p} 2}$ : rate constant of spontaneous synthesis by proximity-controlled concerted metathesis $\mathrm{S}_{\mathrm{a} 1}$ : rate constant of autocatalytic synthesis by proximity-controlled radical substitution $\mathrm{S}_{\mathrm{a} 2}$ : rate constant of autocatalytic synthesis by proximity-controlled concerted metathesis b: rate constant of dimers break down via diffusion-controlled radical substitution 
Supplementary Table 3. Characterisation of the glutathione-protected monomers.

\begin{tabular}{|c|c|c|c|c|c|}
\hline \multirow{2}{*}{ Compounds } & \multirow{2}{*}{$\begin{array}{c}\text { Exact } \\
\text { mass (Da) }\end{array}$} & \multicolumn{2}{|c|}{ Detected ions } & Retention time & Retention time \\
& {$\left[\mathrm{M}+1 \mathrm{H}^{+}\right]^{1+}$} & {$\left[\mathrm{M}+2 \mathrm{H}^{+}\right]^{2+}$} & $(\mathrm{min})^{*}$ & $(\mathrm{~min}) * *$ \\
\hline IF-SG & 1327.62 & 1328.67 & 664.84 & 20.81 & 14.49 \\
\hline KW-SG & 1381.69 & 1382.69 & 691.85 & 16.58 & 10.42 \\
\hline LW-SG & 1366.68 & 1367.68 & 684.35 & 21.79 & 14.76 \\
\hline QW-SG & 1381.65 & 1382.65 & 691.83 & 17.48 & 11.60 \\
\hline RF-SG & 1370.68 & 1371.69 & 686.35 & 16.62 & 10.31 \\
\hline RW-SG & 1409.69 & 1410.7 & 705.85 & 18.03 & 10.56 \\
\hline SW-SG & 1340.63 & 1341.63 & 671.32 & 17.23 & 11.60 \\
\hline TW-SG & 1354.64 & 1355.65 & 678.33 & 19.15 & 12.06 \\
\hline VW-SG & 1352.66 & 1353.67 & 677.34 & 20.11 & 14.05 \\
\hline WF-SG & 1400.66 & 1401.67 & 701.34 & 21.37 & 14.29 \\
\hline WW-SG & 1439.67 & 1440.68 & 720.84 & 17.23 & 12.86 \\
\hline YF-SG & 1377.66 & 1378.65 & 689.83 & 18.94 & 12.98 \\
\hline
\end{tabular}

*Analytical HPLC-UV measurement. Column: Phenomenex Luna C18 $(250 \times 4.6 \mathrm{~mm})$. Method: $5-80 \%$ B during 25 min, flow rate: $1.2 \mathrm{~mL} \mathrm{~min}^{-1}$, where eluent A: $0.1 \%$ TFA in water, eluent B: $0.1 \%$ TFA and $80 \% \mathrm{ACN}$ in water.

**Analytical HPLC-MS measurement. Column: Aeris Widepore XB-C18 $(250 \times 4.6 \mathrm{~mm})$. Method: 5-80\% $\mathrm{B}$ during $25 \mathrm{~min}$, flow rate: $0.7 \mathrm{~mL} \mathrm{~min}^{-1}$, where eluent A: $0.1 \% \mathrm{HCOOH}$ in water, eluent B: $0.1 \% \mathrm{HCOOH}$ in ACN.

Supplementary Table 4. Calibration of the MS Area Under Curve (AUC) to concentration conversion for each component of the system.

\begin{tabular}{|c|c|c|c|c|}
\hline compounds & $\begin{array}{c}\text { calculated } \\
\text { concentration } \\
(\mu \mathrm{M})^{*}\end{array}$ & mean AUC & SD & $\begin{array}{c}\text { Conversion } \\
\text { factor: } \\
\text { AUC/ } \mu \mathrm{M} \\
\end{array}$ \\
\hline IF-IF & 0.2083 & 6738896 & 167227 & 32346754 \\
\hline IF-QW & 0.4167 & 14321621 & 1021416 & 34371864 \\
\hline IF-SG & 5 & 233796364 & 12121312 & 46759273 \\
\hline IF-SW & 0.4167 & 10250563 & 267887 & 24601332 \\
\hline IF-TW & 0.4167 & 9781464 & 99474 & 23475495 \\
\hline IF-VW & 0.4167 & 14658581 & 333948 & 35180567 \\
\hline KW-IF & 0.4167 & 6984610 & 192959 & 16763051 \\
\hline KW-KW & 0.2083 & 4688541 & 196654 & 22505032 \\
\hline KW-LW & 0.4167 & 10931447 & 352192 & 26235452 \\
\hline KW-QW & 0.4167 & 9592254 & 191099 & 23021391 \\
\hline KW-RF & 0.4167 & 6041711 & 280144 & 14500094 \\
\hline KW-SG & 5 & 93498264 & 8075947 & 18699653 \\
\hline KW-SW & 0.4167 & 10307687 & 34078 & 24738429 \\
\hline KW-TW & 0.4167 & 11140758 & 215090 & 26737798 \\
\hline
\end{tabular}




\begin{tabular}{|c|c|c|c|c|}
\hline KW-VW & 0.4167 & 11219383 & 61459 & 26926498 \\
\hline LW-IF & 0.4167 & 13317251 & 59335 & 31961377 \\
\hline LW-LW & 0.2083 & 5459491 & 232775 & 26205601 \\
\hline LW-QW & 0.4167 & 7510246 & 171240 & 18024575 \\
\hline LW-SG & 5 & 71564374 & 1943793 & 14312875 \\
\hline LW-SW & 0.4167 & 7846422 & 820359 & 18831398 \\
\hline LW-TW & 0.4167 & 7877221 & 1158047 & 18905315 \\
\hline LW-VW & 0.4167 & 11755384 & 350708 & 28212900 \\
\hline QW-QW & 0.2083 & 2505601 & 113767 & 12026904 \\
\hline QW-SG & 5 & 154788096 & 3212397 & 30957619 \\
\hline RF-IF & 0.4167 & 9685531 & 98651 & 23245255 \\
\hline RF-LW & 0.4167 & 9423472 & 313860 & 22616315 \\
\hline RF-QW & 0.4167 & 5395324 & 470128 & 12948767 \\
\hline RF-RF & 0.2083 & 2600196 & 66430 & 12480959 \\
\hline RF-SG & 5 & 76355793 & 557309 & 15271159 \\
\hline RF-SW & 0.4167 & 8404764 & 40877 & 20171417 \\
\hline RF-TW & 0.4167 & 6458569 & 88664 & 15500554 \\
\hline RF-VW & 0.4167 & 9015525 & 232567 & 21637242 \\
\hline RW-IF & 0.4167 & 9139403 & 235650 & 21934550 \\
\hline RW-KW & 0.4167 & 7268733 & 135533 & 17444946 \\
\hline RW-LW & 0.4167 & 9690533 & 135870 & 23257261 \\
\hline RW-QW & 0.4167 & 8128642 & 168034 & 19508725 \\
\hline RW-RF & 0.4167 & 4839118 & 14804 & 11613873 \\
\hline RW-RW & 0.2083 & 2840665 & 146521 & 13635212 \\
\hline RW-SG & 5 & 81307536 & 3537250 & 16261507 \\
\hline RW-SW & 0.4167 & 9262117 & 211359 & 22229063 \\
\hline RW-TW & 0.4167 & 5594796 & 220034 & 13427499 \\
\hline RW-VW & 0.4167 & 10268484 & 436026 & 24644341 \\
\hline SW-QW & 0.4167 & 7741284 & 52795 & 18579067 \\
\hline SW-SG & 5 & 214373675 & 971923 & 42874735 \\
\hline SW-SW & 0.2083 & 4460048 & 9425 & 21408267 \\
\hline TW-QW & 0.4167 & 6258758 & 7940 & 15021007 \\
\hline TW-SG & 5 & 205650252 & 4735772 & 41130050 \\
\hline TW-SW & 0.4167 & 7866053 & 65835 & 18878512 \\
\hline TW-TW & 0.2083 & 6984610 & 192959 & 33526183 \\
\hline VW-QW & 0.4167 & 8395542 & 149336 & 20149285 \\
\hline VW-SG & 5 & 218439667 & 209807 & 43687933 \\
\hline VW-SW & 0.4167 & 9549648 & 30622 & 22919137 \\
\hline VW-TW & 0.4167 & 14321621 & 1021416 & 34371864 \\
\hline VW-VW & 0.2083 & 6719439 & 16227 & 32253357 \\
\hline WF-IF & 0.4167 & 16802806 & 261057 & 40326702 \\
\hline WF-KW & 0.4167 & 12948964 & 294491 & 31077488 \\
\hline WF-LW & 0.4167 & 14057330 & 138666 & 33737565 \\
\hline
\end{tabular}




\begin{tabular}{|c|r|r|r|r|}
\hline WF-QW & 0.4167 & 9308290 & 140062 & $\mathbf{2 2 3 3 9 8 7 8}$ \\
\hline WF-RF & 0.4167 & 9750493 & 108676 & $\mathbf{2 3 4 0 1 1 6 3}$ \\
\hline WF-RW & 0.4167 & 5231109 & 21871 & $\mathbf{1 2 5 5 4 6 5 2}$ \\
\hline WF-SG & 5 & 229629674 & 1234010 & $\mathbf{4 5 9 2 5 9 3 5}$ \\
\hline WF-SW & 0.4167 & 10201405 & 81613 & $\mathbf{2 4 4 8 3 3 5 2}$ \\
\hline WF-TW & 0.4167 & 10261229 & 335150 & $\mathbf{2 4 6 2 6 9 3 0}$ \\
\hline WF-VW & 0.4167 & 12943413 & 2777026 & $\mathbf{3 1 0 6 4 1 6 7}$ \\
\hline WF-WF & 0.2083 & 9182636 & 141237 & $\mathbf{4 4 0 7 6 7 2 2}$ \\
\hline WF-WW & 0.4167 & 7037382 & 357237 & $\mathbf{1 6 8 8 9 7 0 4}$ \\
\hline WF-YF & 0.4167 & 11977596 & 184803 & $\mathbf{2 8 7 4 6 2 0 8}$ \\
\hline WW-IF & 0.4167 & 6697741 & 203025 & $\mathbf{1 6 0 7 4 5 6 6}$ \\
\hline WW-KW & 0.4167 & 7218262 & 137235 & $\mathbf{1 7 3 2 3 8 1 6}$ \\
\hline WW-LW & 0.4167 & 6808518 & 206582 & $\mathbf{1 6 3 4 0 4 3 0}$ \\
\hline WW-QW & 0.4167 & 4956501 & 97189 & $\mathbf{1 1 8 9 5 5 9 2}$ \\
\hline WW-RF & 0.4167 & 5597430 & 97043 & $\mathbf{1 3 4 3 3 8 2 2}$ \\
\hline WW-RW & 0.4167 & 5744039 & 55944 & $\mathbf{1 3 7 8 5 6 8 3}$ \\
\hline WW-SG & 5 & 154424106 & 809265 & $\mathbf{3 0 8 8 4 8 2 1}$ \\
\hline WW-SW & 0.4167 & 7736738 & 259061 & $\mathbf{1 8 5 6 8 1 5 8}$ \\
\hline WW-TW & 0.4167 & 6666086 & 237986 & $\mathbf{1 5 9 9 8 5 9 3}$ \\
\hline WW-VW & 0.4167 & 6666086 & 237986 & $\mathbf{1 5 9 9 8 5 9 3}$ \\
\hline WW-WW & 0.2083 & 2408501 & 253201 & $\mathbf{1 1 5 6 0 8 2 2}$ \\
\hline WW-YF & 0.4167 & 4863880 & 87381 & $\mathbf{1 1 6 7 3 3 0 2}$ \\
\hline YF-IF & 0.4167 & 8352642 & 1717484 & $\mathbf{2 0 0 4 6 3 2 5}$ \\
\hline YF-KW & 0.4167 & 8037313 & 69720 & $\mathbf{1 9 2 8 9 5 3 5}$ \\
\hline YF-LW & 0.4167 & 9072627 & 56896 & $\mathbf{2 1 7 7 4 2 8 6}$ \\
\hline YF-QW & 0.4167 & 6032798 & 175591 & $\mathbf{1 4 4 7 8 7 0 3}$ \\
\hline YF-RF & 0.4167 & 6941704 & 43753 & $\mathbf{1 6 6 6 0 0 7 7}$ \\
\hline YF-RW & 0.4167 & 7672105 & 108675 & $\mathbf{1 8 4 1 3 0 3 7}$ \\
\hline YF-SG & 5 & 177069749 & 1736459 & $\mathbf{3 5 4 1 3 9 5 0}$ \\
\hline YF-SW & 0.4167 & 6309200 & 101256 & $\mathbf{1 5 1 4 2 0 6 9}$ \\
\hline YF-TW & 0.4167 & 6577118 & 44472 & $\mathbf{1 5 7 8 5 0 7 1}$ \\
\hline YF-VW & 0.4167 & 10338711 & 140452 & $\mathbf{2 4 8 1 2 8 8 7}$ \\
\hline YF-YF & 0.2083 & 2466209 & 13733 & $\mathbf{1 1 8 3 7 8 2 5}$ \\
\hline
\end{tabular}

$*$ Calculated from the statistical product distribution 


\section{Peptide characterisation data}

HPLC and MS characterization of the individual monomeric sequences
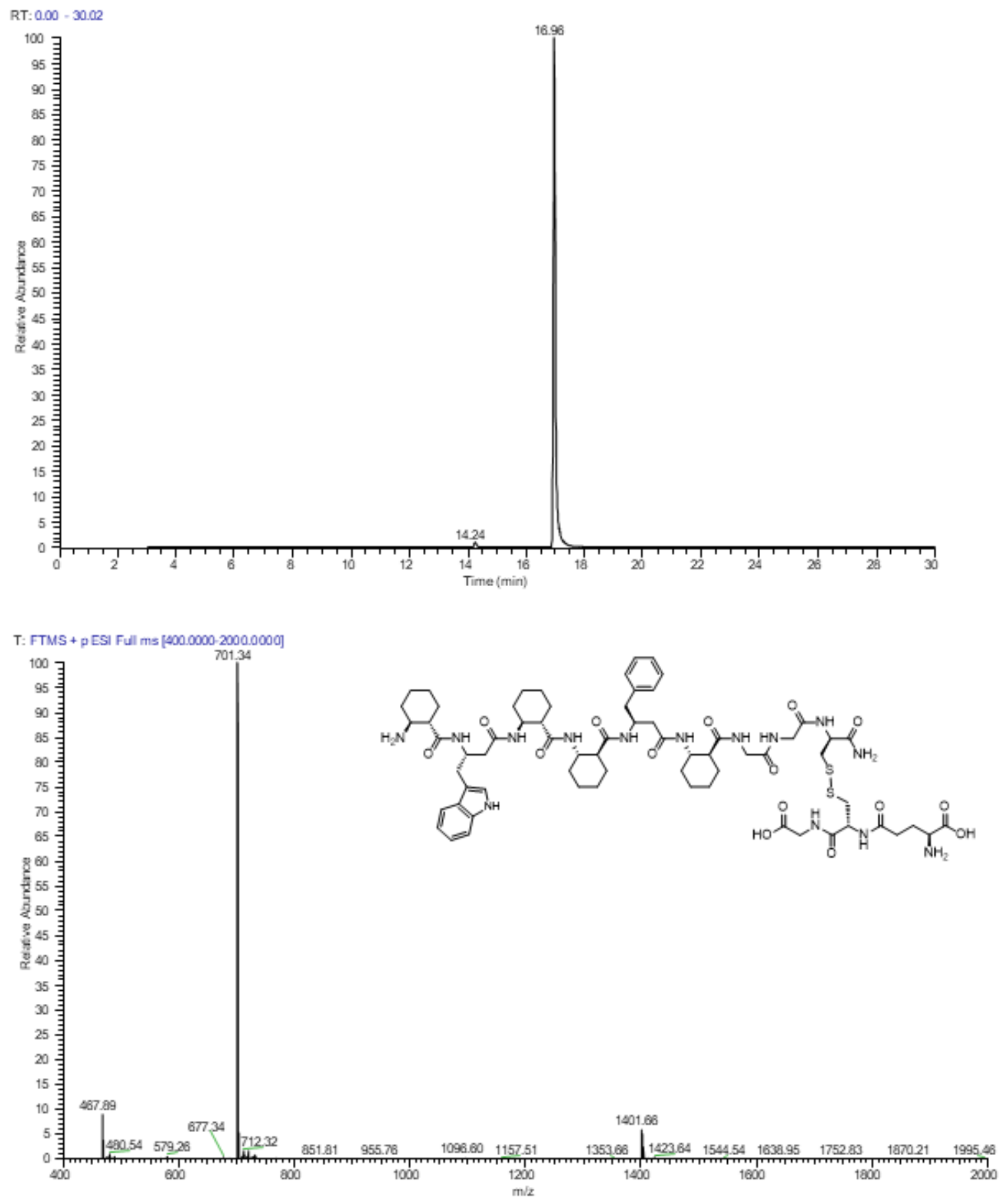

HPLC trace and mass spectrum of the glutathionic precursor WF-SG (exact mass: 1400.66). Calculated isotopic profile for $[\mathrm{M}+2 \mathrm{H}]^{2+}$ (species, abundance): $701.3383(100 \%), 701.8400$ (72.47\%), $702.3417(25.86 \%), 702.8379$ (6.48\%); m/z calculated: $1401.67[\mathrm{M}+\mathrm{H}]^{+}, 701.24$ $[\mathrm{M}+2 \mathrm{H}]^{2+} ; \mathrm{m} / \mathrm{z}$ observed: $1401.06[\mathrm{M}+\mathrm{H}]^{+}, 701.34[\mathrm{M}+2 \mathrm{H}]^{2+}$ 

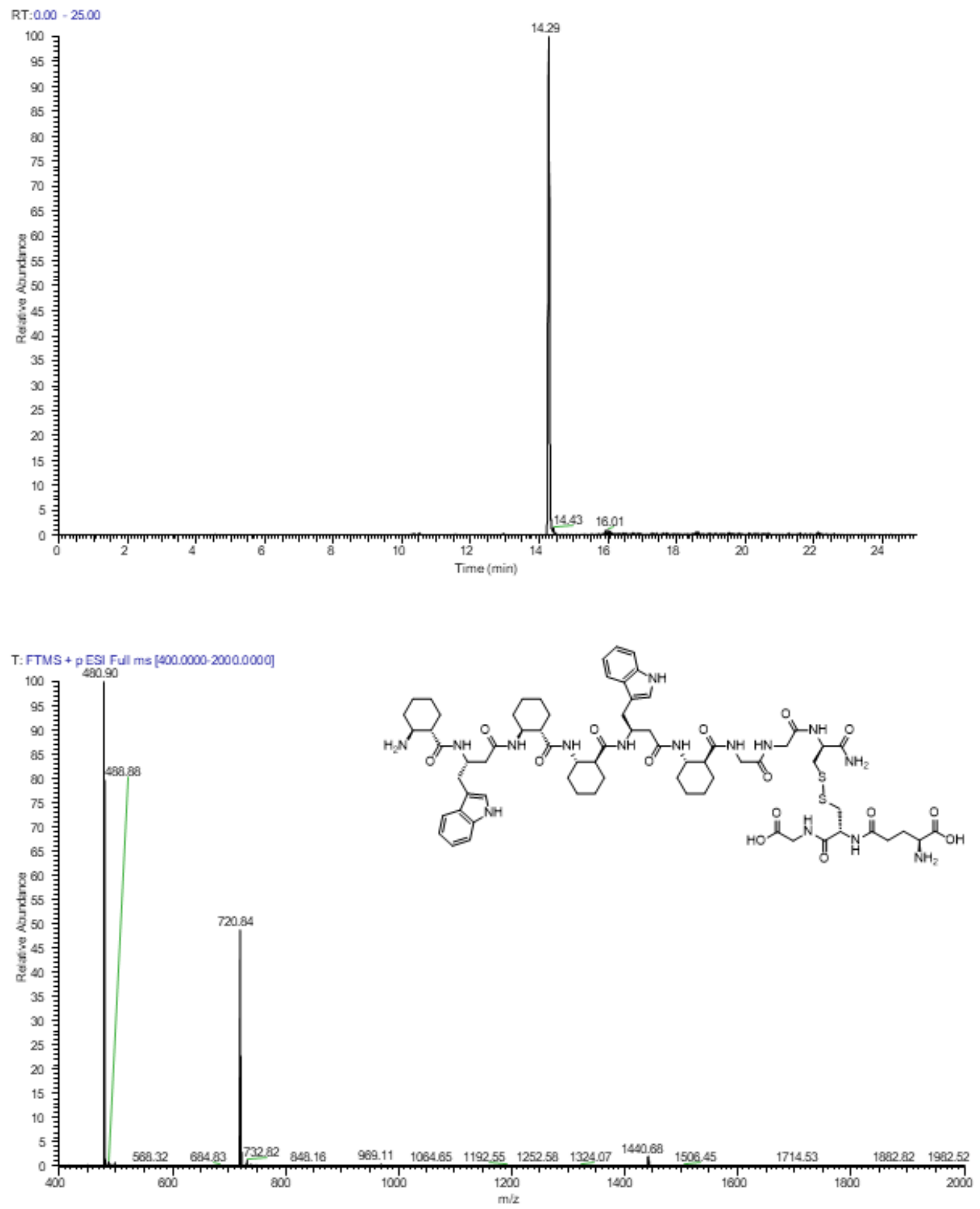

HPLC trace and mass spectrum of the glutathionic precursor WW-SG (exact mass: 1439.67). Calculated isotopic profile for $[\mathrm{M}+2 \mathrm{H}] 2+$ (species, abundance): 720.8438 (100\%), 721.3454 (74.63\%), 721.8471 (27.44\%), $722.3434(6.78 \%) ; \mathrm{m} / \mathrm{z}$ calculated: $1440.51[\mathrm{M}+\mathrm{H}]^{+}, 720.76$ $[\mathrm{M}+2 \mathrm{H}]^{2+}, 480.84[\mathrm{M}+3 \mathrm{H}]^{3+} ; \mathrm{m} / \mathrm{z}$ observed: $1440.68[\mathrm{M}+\mathrm{H}]^{+}, 720.64[\mathrm{M}+2 \mathrm{H}]^{2+}, 480.90$ $[\mathrm{M}+3 \mathrm{H}]^{3+}$ 

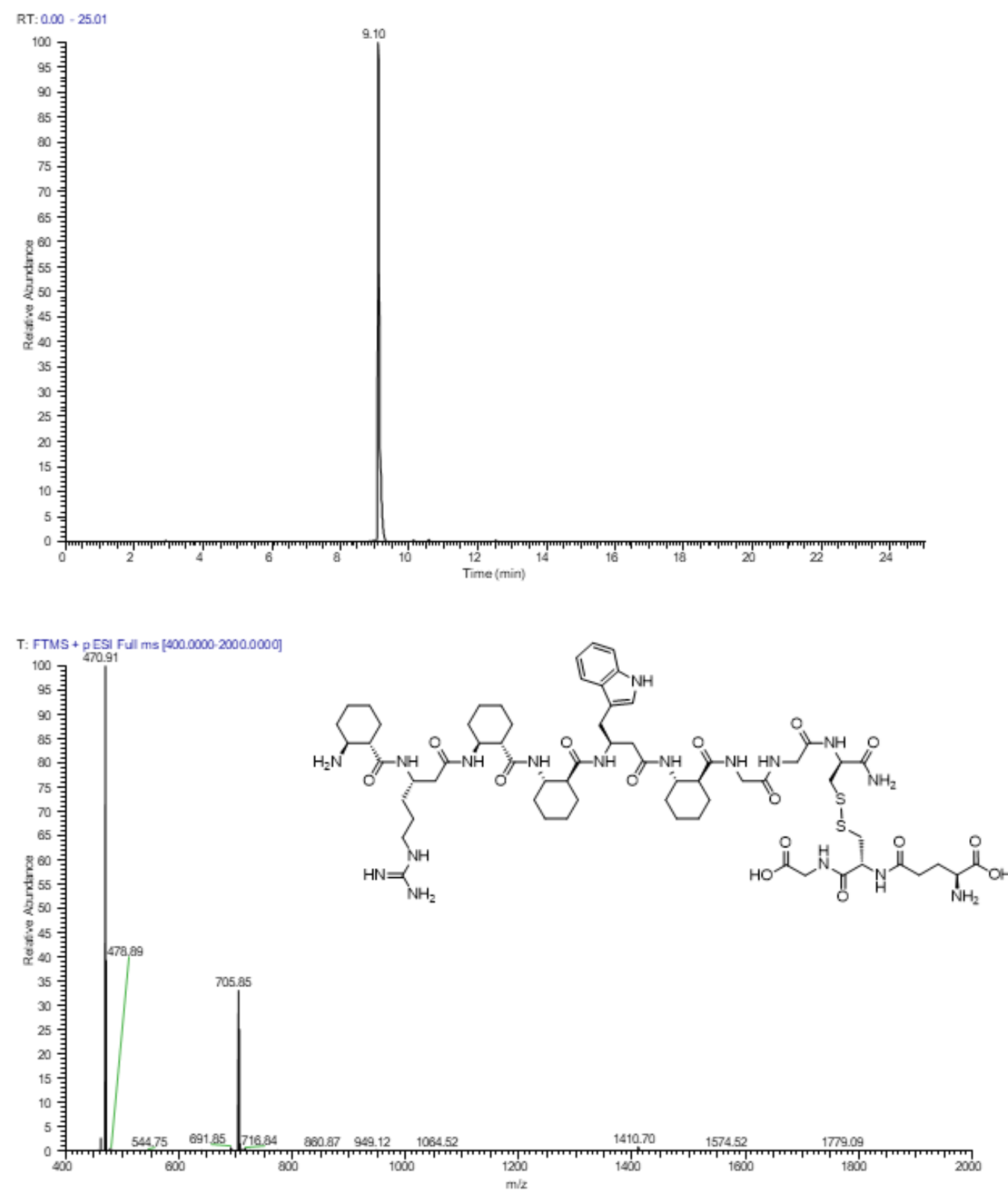

HPLC trace and mass spectrum of the glutathionic precursor RW-SG (exact mass: 1409.69). Calculated isotopic profile for $[\mathrm{M}+2 \mathrm{H}]^{2+}$ (species, abundance): 705.3508 (100\%), 705.8524 (69.22\%), 706.3487 (8.95\%), 706.3510 (4.30\%); m/z calculated: $1410.49[\mathrm{M}+\mathrm{H}]^{+}, 705.75$ $[\mathrm{M}+2 \mathrm{H}]^{2+}, 470.83[\mathrm{M}+3 \mathrm{H}]^{3+} ; \mathrm{m} / \mathrm{z}$ observed: $1410.70[\mathrm{M}+\mathrm{H}]^{+}, 705.85[\mathrm{M}+2 \mathrm{H}]^{2+}, 470.91$ $[\mathrm{M}+3 \mathrm{H}]^{3+}$ 

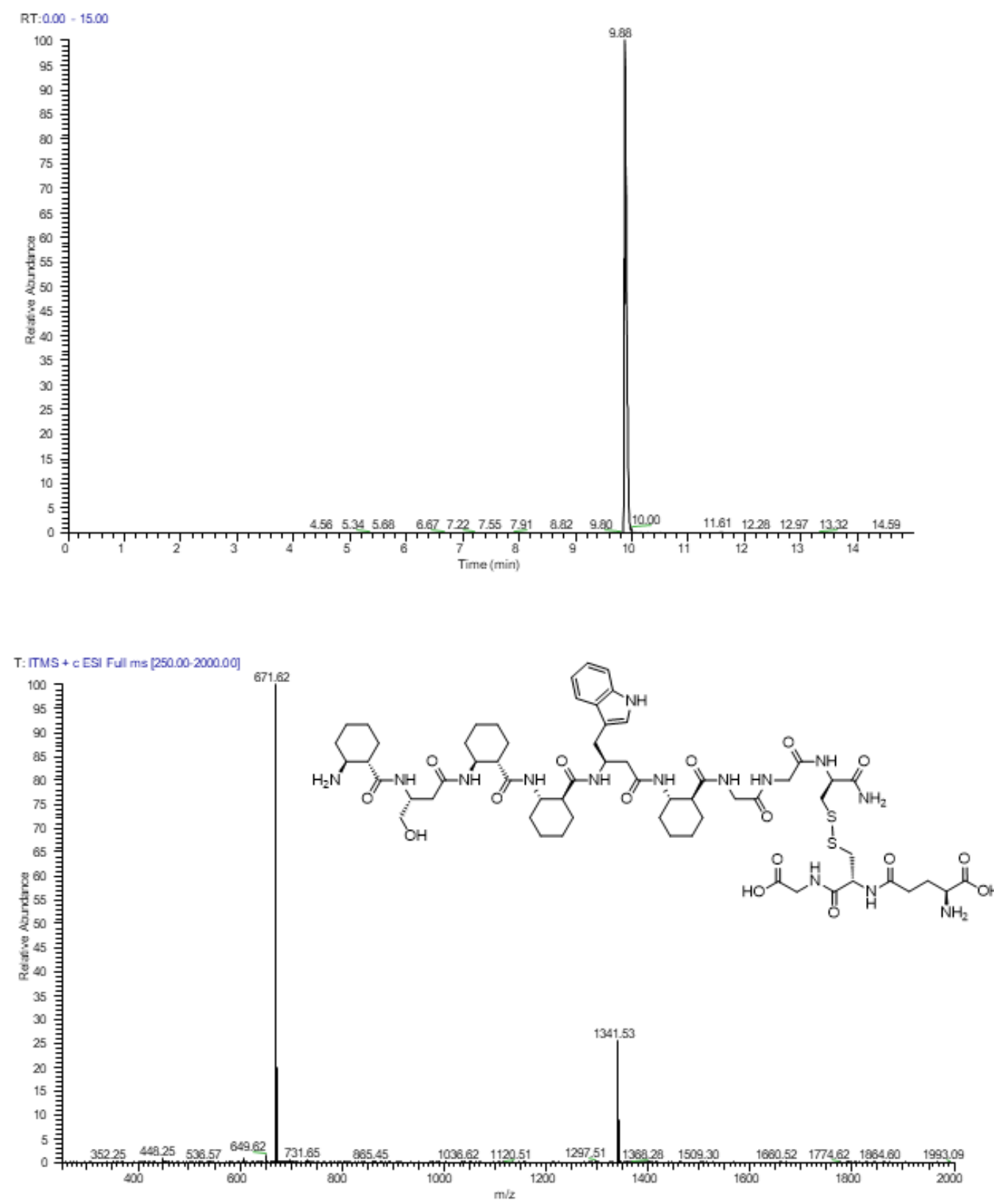

HPLC trace and mass spectrum of the glutathionic precursor SW-SG (exact mass: 1340.63). Calculated isotopic profile for $[\mathrm{M}+2 \mathrm{H}]^{2+}$ (species, abundance): 670.8162 (100\%), 671.3179 (65.98\%), 671.8141 (8.95\%), 671.8164 (3.37\%); m/z calculated: $1341.38[\mathrm{M}+\mathrm{H}]^{+}, 671.19$ $[\mathrm{M}+2 \mathrm{H}]^{2+} ; \mathrm{m} / \mathrm{z}$ observed: $1341.53[\mathrm{M}+\mathrm{H}]^{+}, 671.62[\mathrm{M}+2 \mathrm{H}]^{2+}$ 

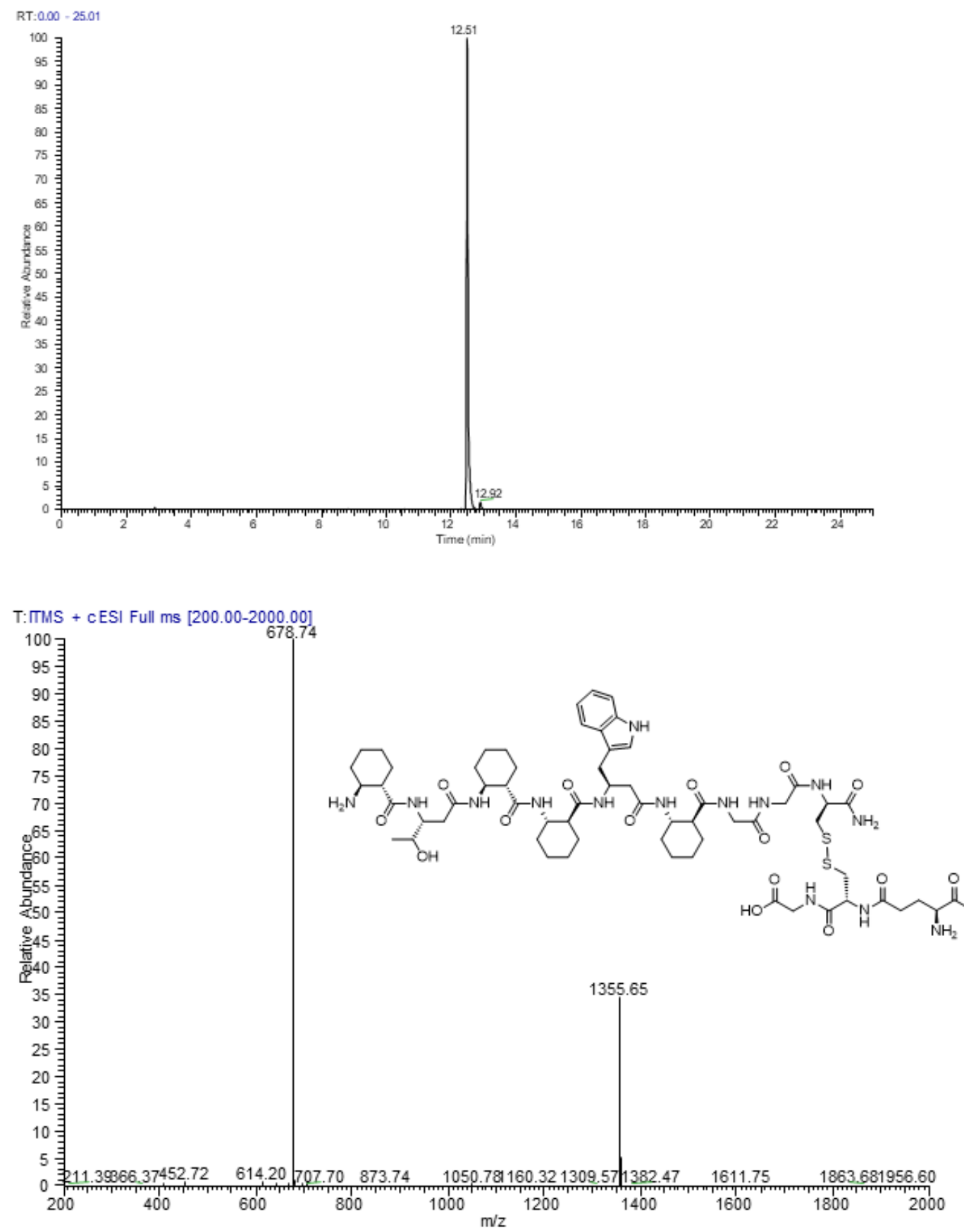

HPLC trace and mass spectrum of the glutathionic precursor TW-SG (exact mass: 1354.64). Calculated isotopic profile for $[\mathrm{M}+2 \mathrm{H}]^{2+}$ (species, abundance): 677.8240 (100\%), 678.3257 $(67.06 \%), 678.8211(8.95 \%), 678.8242(3.43 \%) ; \mathrm{m} / \mathrm{z}$ calculated: $1355.41[\mathrm{M}+\mathrm{H}]^{+}, 678.21$ $[\mathrm{M}+2 \mathrm{H}]^{2+} ; \mathrm{m} / \mathrm{z}$ observed: $1355.65[\mathrm{M}+\mathrm{H}]^{+}, 678.74[\mathrm{M}+2 \mathrm{H}]^{2+}$ 

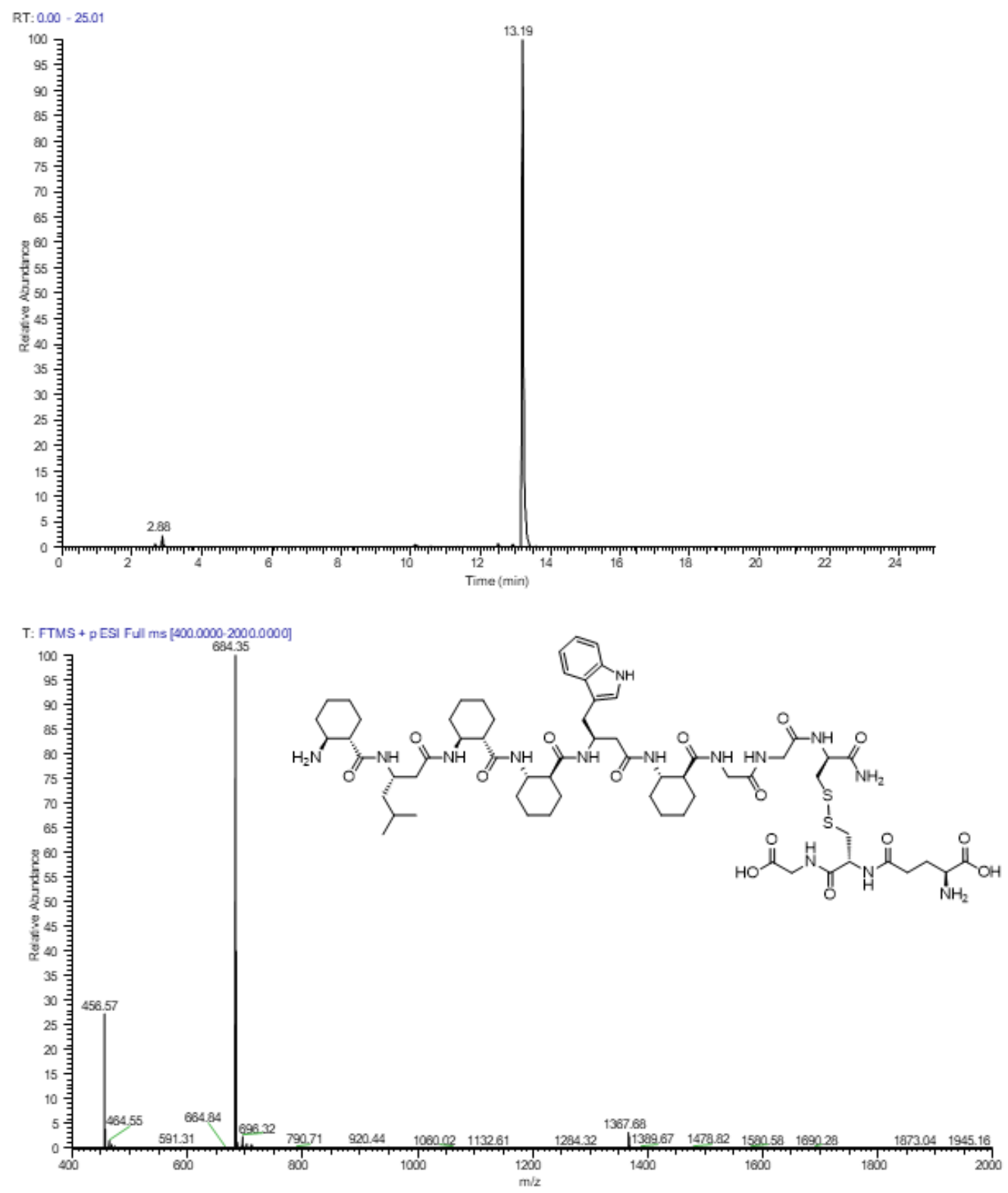

HPLC trace and mass spectrum of the glutathionic precursor LW-SG (exact mass: 1366.68). Calculated isotopic profile for $[\mathrm{M}+2 \mathrm{H}]^{2+}$ (species, abundance): $683.8422(100 \%), 684.3439$ (69.22\%), $684.8401(8.95 \%), 684.8424(3.54 \%) ; \mathrm{m} / \mathrm{z}$ calculated: $1367.46[\mathrm{M}+\mathrm{H}]^{+}, 684.23$ $[\mathrm{M}+2 \mathrm{H}]^{2+} ; \mathrm{m} / \mathrm{z}$ observed: $1367.68[\mathrm{M}+\mathrm{H}]^{+}, 684.35[\mathrm{M}+2 \mathrm{H}]^{2+}$ 

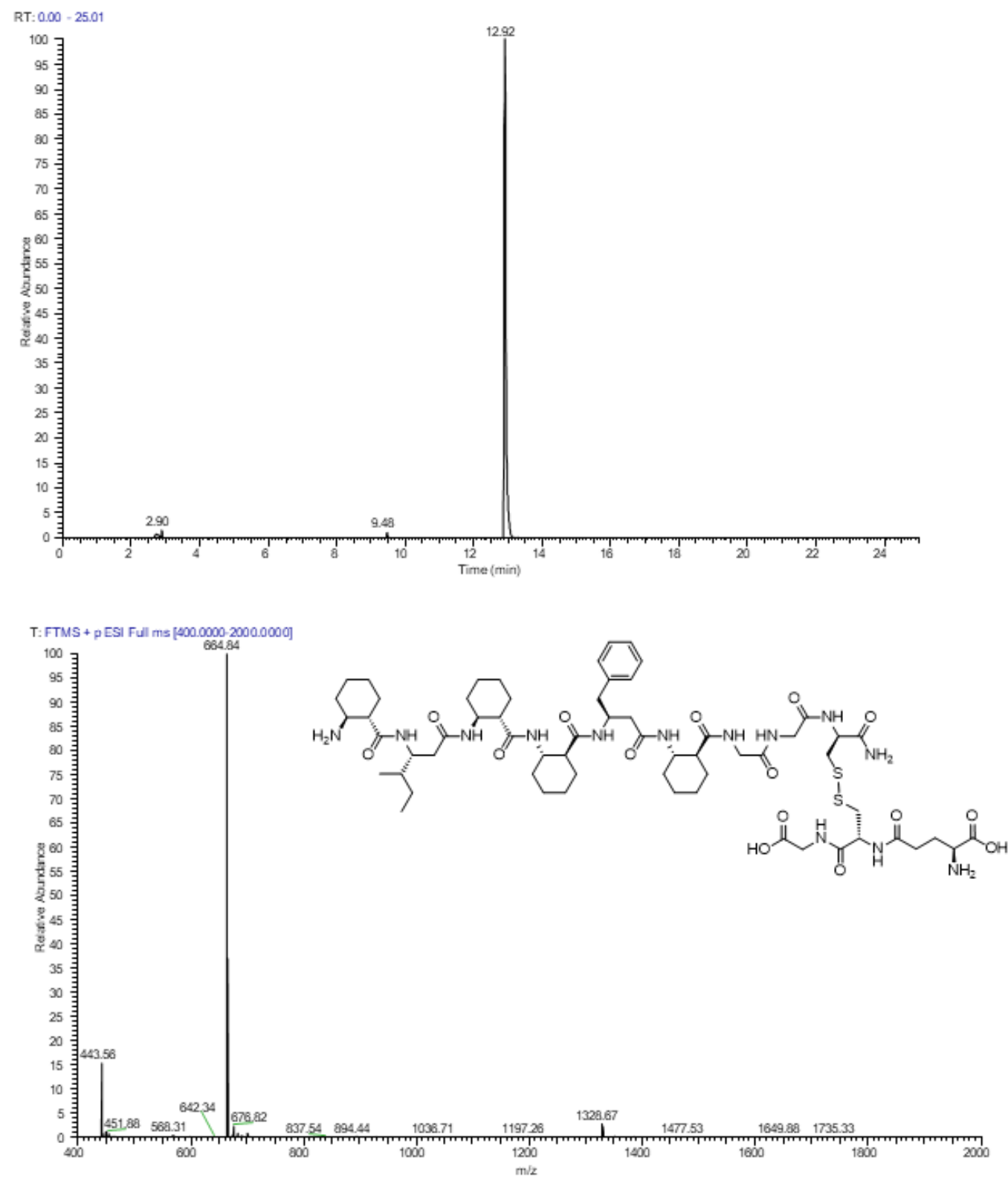

HPLC trace and mass spectrum of the glutathionic precursor IF-SG (exact mass: 1327.67). Calculated isotopic profile for $[\mathrm{M}+2 \mathrm{H}]^{2+}$ (species, abundance): 664.3468 (100\%), 664.8385 (67.06\%), 665.3347 (8.95\%), 665.3370 (3.18\%); m/z calculated: $1328.42[\mathrm{M}+\mathrm{H}]^{+}, 664.71$ $[\mathrm{M}+2 \mathrm{H}]^{2+} ; \mathrm{m} / \mathrm{z}$ observed: $1328.67[\mathrm{M}+\mathrm{H}]^{+}, 664.84[\mathrm{M}+2 \mathrm{H}]^{2+}$ 

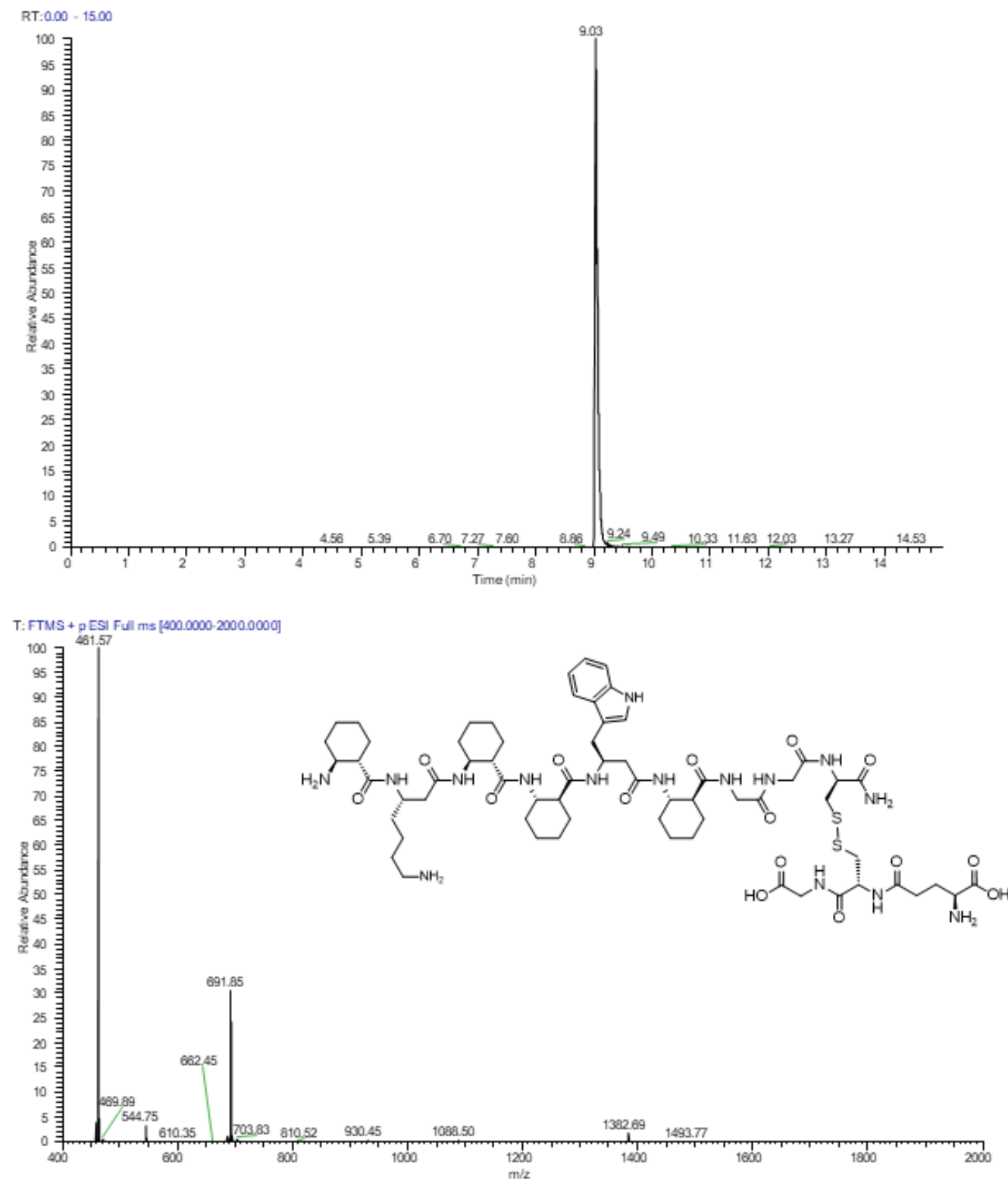

HPLC trace and mass spectrum of the glutathionic precursor KW-SG (exact mass: 1381.69). Calculated isotopic profile for $[\mathrm{M}+2 \mathrm{H}]^{2+}$ (species, abundance): 691.3477 (100\%), 691.8494 (69.22\%), $692.3447(8.95 \%), 692.3479$ (3.79\%); m/z calculated: $1382.47[\mathrm{M}+\mathrm{H}]^{+}, 691.74$ $[\mathrm{M}+2 \mathrm{H}]^{2+}, 461.49[\mathrm{M}+3 \mathrm{H}]^{3+} ; \mathrm{m} / \mathrm{z}$ observed: $1382.69[\mathrm{M}+\mathrm{H}]^{+}, 691.85[\mathrm{M}+2 \mathrm{H}]^{2+}, 461.57$ $[\mathrm{M}+3 \mathrm{H}]^{3+}$ 

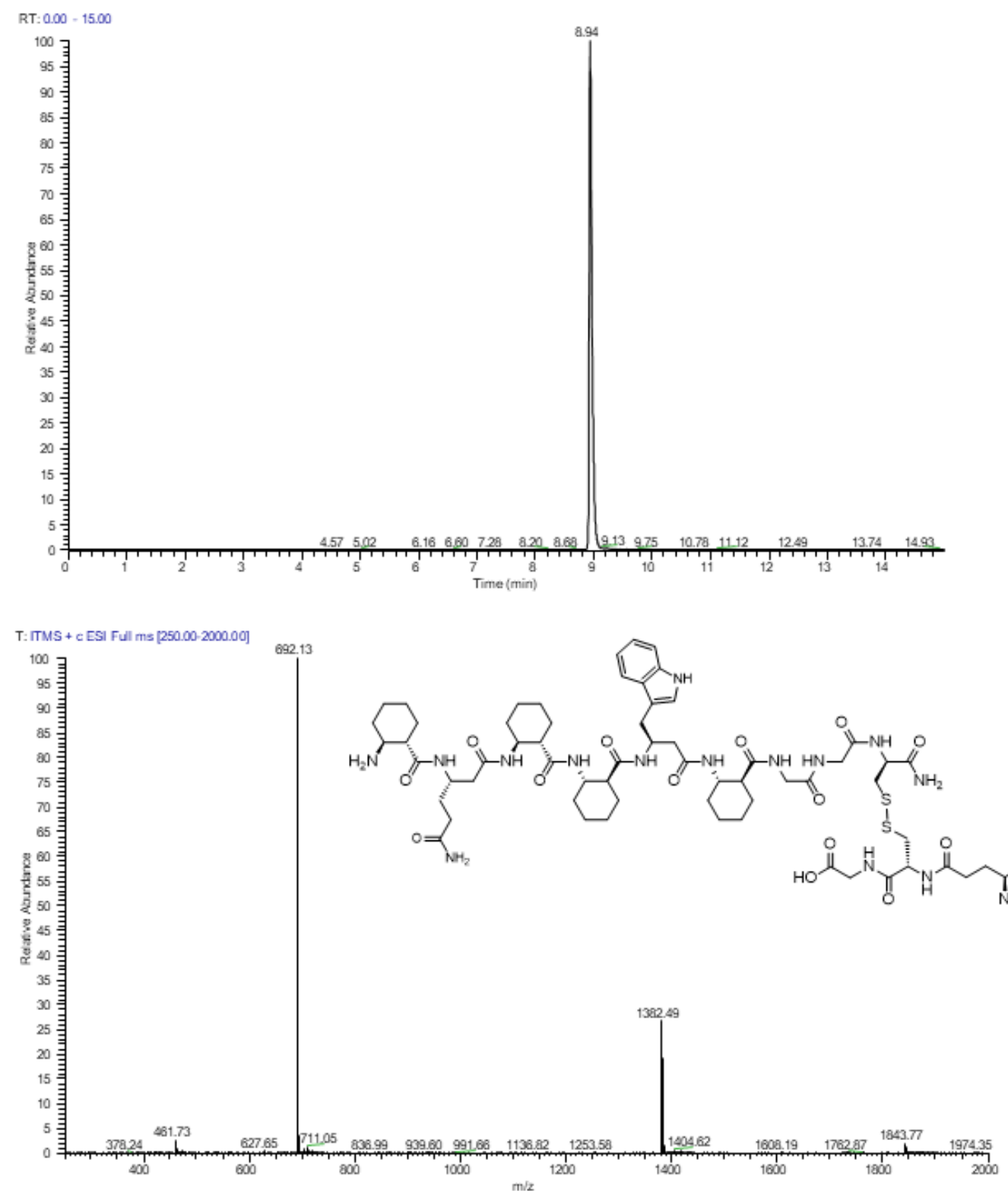

HPLC trace and mass spectrum of the glutathionic precursor QW-SG (exact mass: 1381.65). Calculated isotopic profile for $[\mathrm{M}+2 \mathrm{H}]^{2+}$ (species, abundance): 691.3295 (100\%), 691.8312 (68.14\%), $692.3274(8.95 \%), 692.3297(3.73 \%) ; \mathrm{m} / \mathrm{z}$ calculated: $1382.43[\mathrm{M}+\mathrm{H}]^{+}, 691.72$ $[\mathrm{M}+2 \mathrm{H}]^{2+} ; \mathrm{m} / \mathrm{z}$ observed: $1382.49[\mathrm{M}+\mathrm{H}]^{+}, 692.13[\mathrm{M}+2 \mathrm{H}]^{2+}$ 

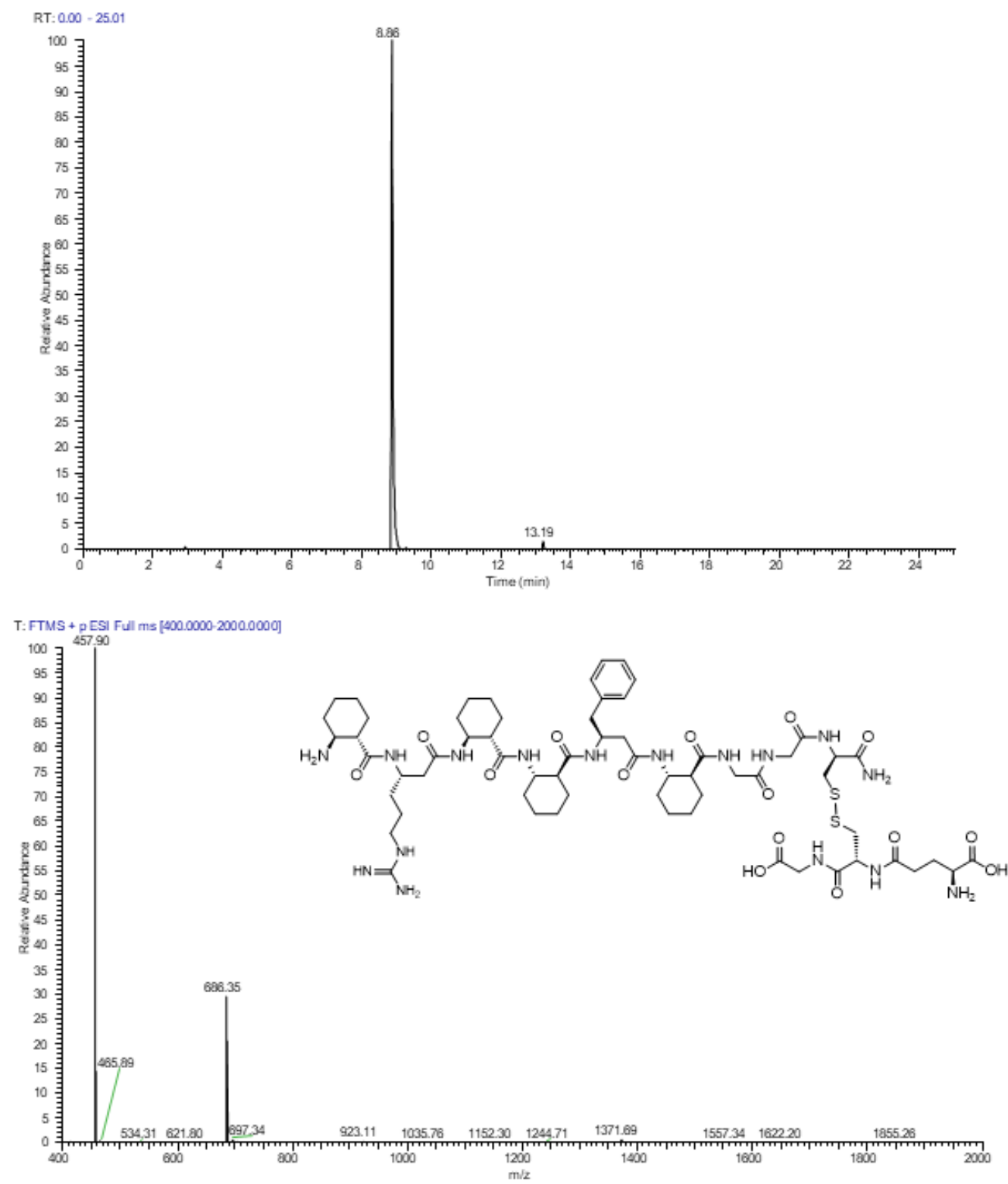

HPLC trace and mass spectrum of the glutathionic precursor RF-SG (exact mass: 1370.68). Calculated isotopic profile for $[\mathrm{M}+2 \mathrm{H}]^{2+}$ (species, abundance): 685.8453 (100\%), 686.3470 $(67.06 \%), 686.8432(8.95 \%), 686.8455(3.92 \%) ; \mathrm{m} / \mathrm{z}$ calculated: $1371.45[\mathrm{M}+\mathrm{H}]^{+}, 686.23$ $[\mathrm{M}+2 \mathrm{H}]^{2+}, 457.82[\mathrm{M}+3 \mathrm{H}]^{3+} ; \mathrm{m} / \mathrm{z}$ observed: $1371.69[\mathrm{M}+\mathrm{H}]^{+}, 686.35[\mathrm{M}+2 \mathrm{H}]^{2+}, 457.90$ $[\mathrm{M}+3 \mathrm{H}]^{3+}$ 

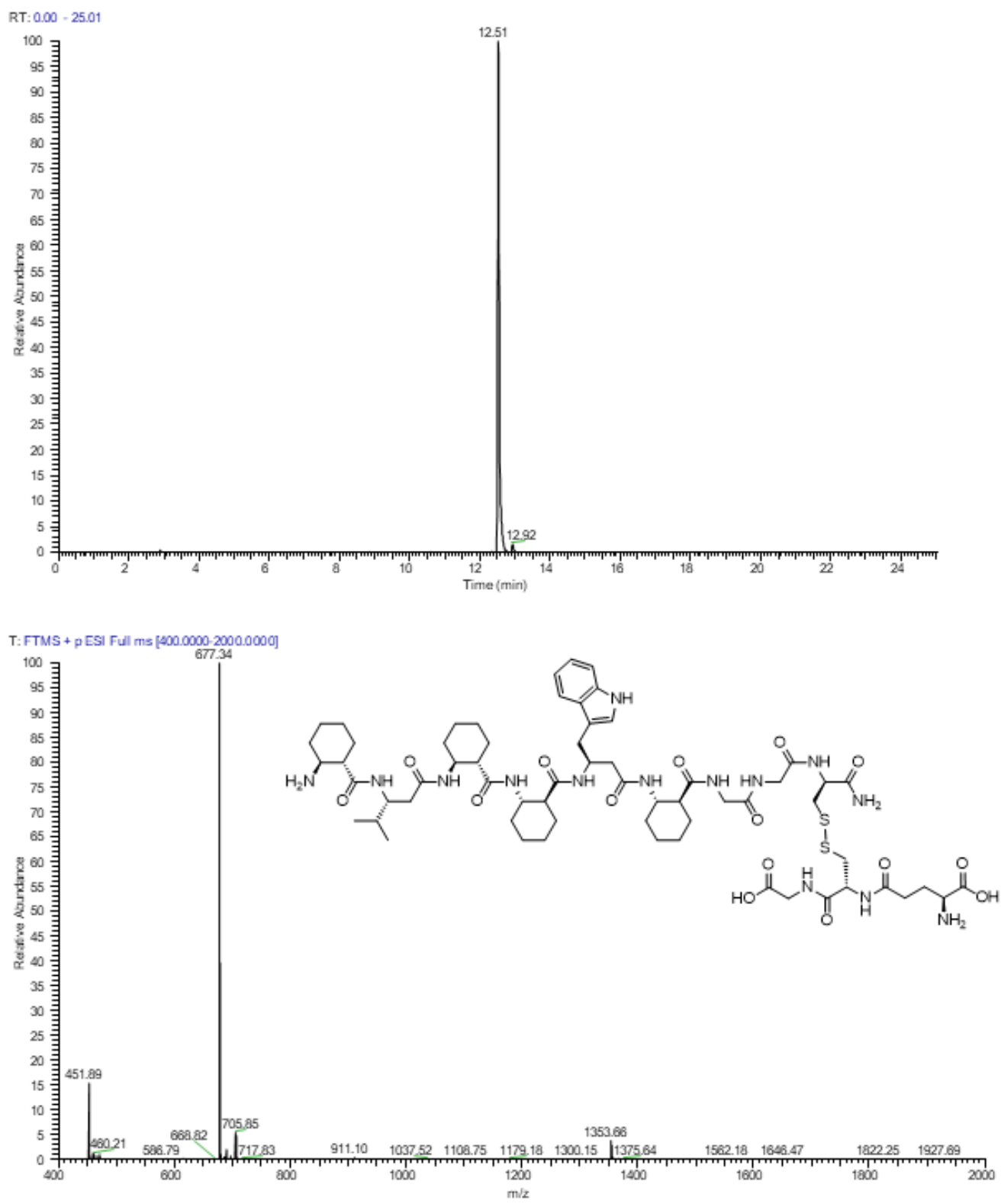

HPLC trace and mass spectrum of the glutathionic precursor VW-SG (exact mass: 1352.66). Calculated isotopic profile for $[\mathrm{M}+2 \mathrm{H}]^{2+}$ (species, abundance): 676.8344 (100\%), 677.3361 (68.14\%), 677.8323 (8.95\%), $677.8346(3.49 \%) ; \mathrm{m} / \mathrm{z}$ calculated: $1353.43[\mathrm{M}+\mathrm{H}]^{+}, 677.22$ $[\mathrm{M}+2 \mathrm{H}]^{2+} ; \mathrm{m} / \mathrm{z}$ observed: $1353.66[\mathrm{M}+\mathrm{H}]^{+}, 677.34[\mathrm{M}+2 \mathrm{H}]^{2+}$ 

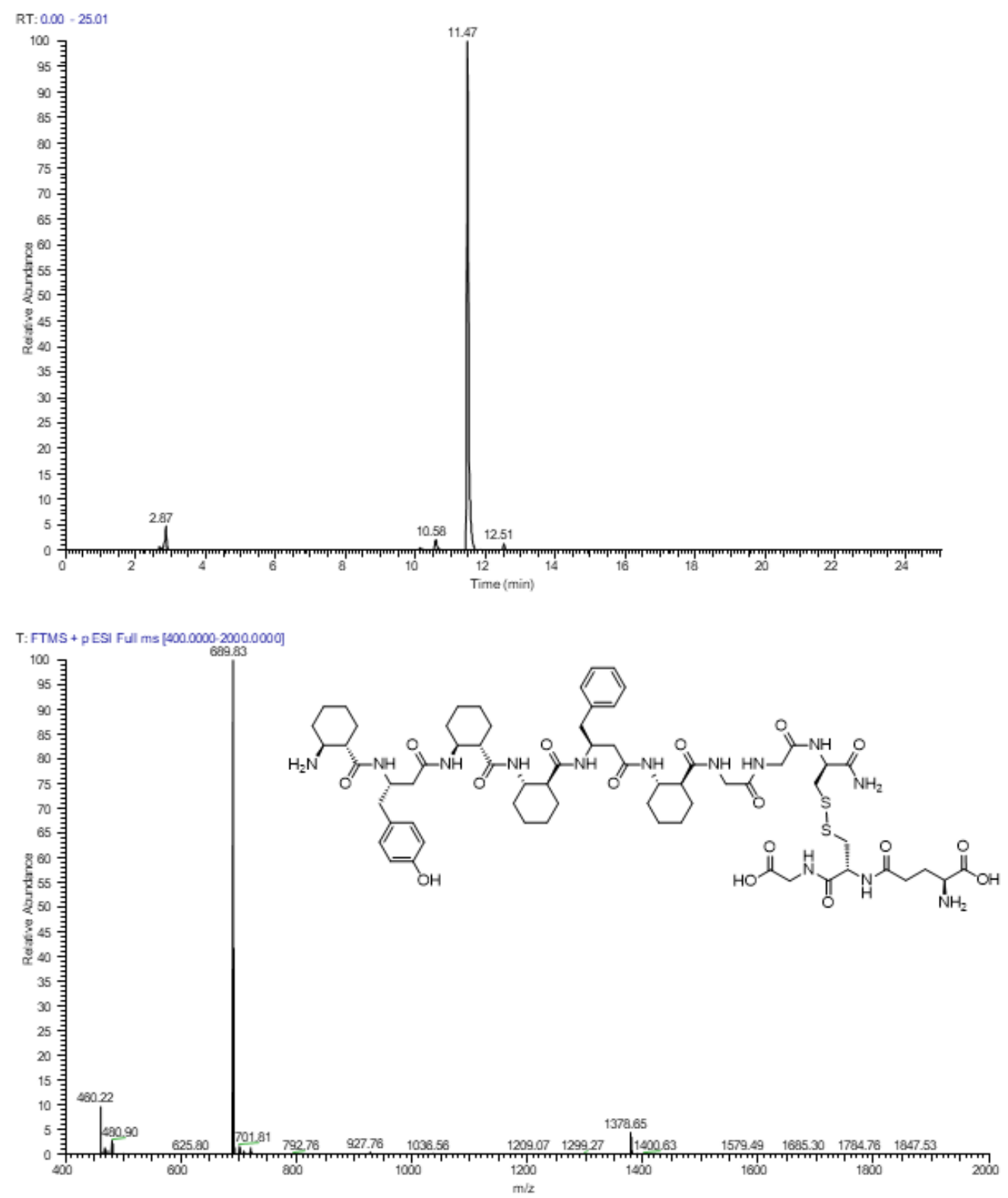

HPLC trace and mass spectrum of the glutathionic precursor YF-SG (exact mass: 1377.65). Calculated isotopic profile for $[\mathrm{M}+2 \mathrm{H}]^{2+}$ (species, abundance): 689.3264 (100\%), 689.8281 (70.30\%), 690.3243 (8.95\%), $690.3266(3.34 \%) ; \mathrm{m} / \mathrm{z}$ calculated: $1378.44[\mathrm{M}+\mathrm{H}]^{+}, 689.72$ $[\mathrm{M}+2 \mathrm{H}]^{2+} ; \mathrm{m} / \mathrm{z}$ observed: $1378.65[\mathrm{M}+\mathrm{H}]^{+}, 689.83[\mathrm{M}+2 \mathrm{H}]^{2+}$ 


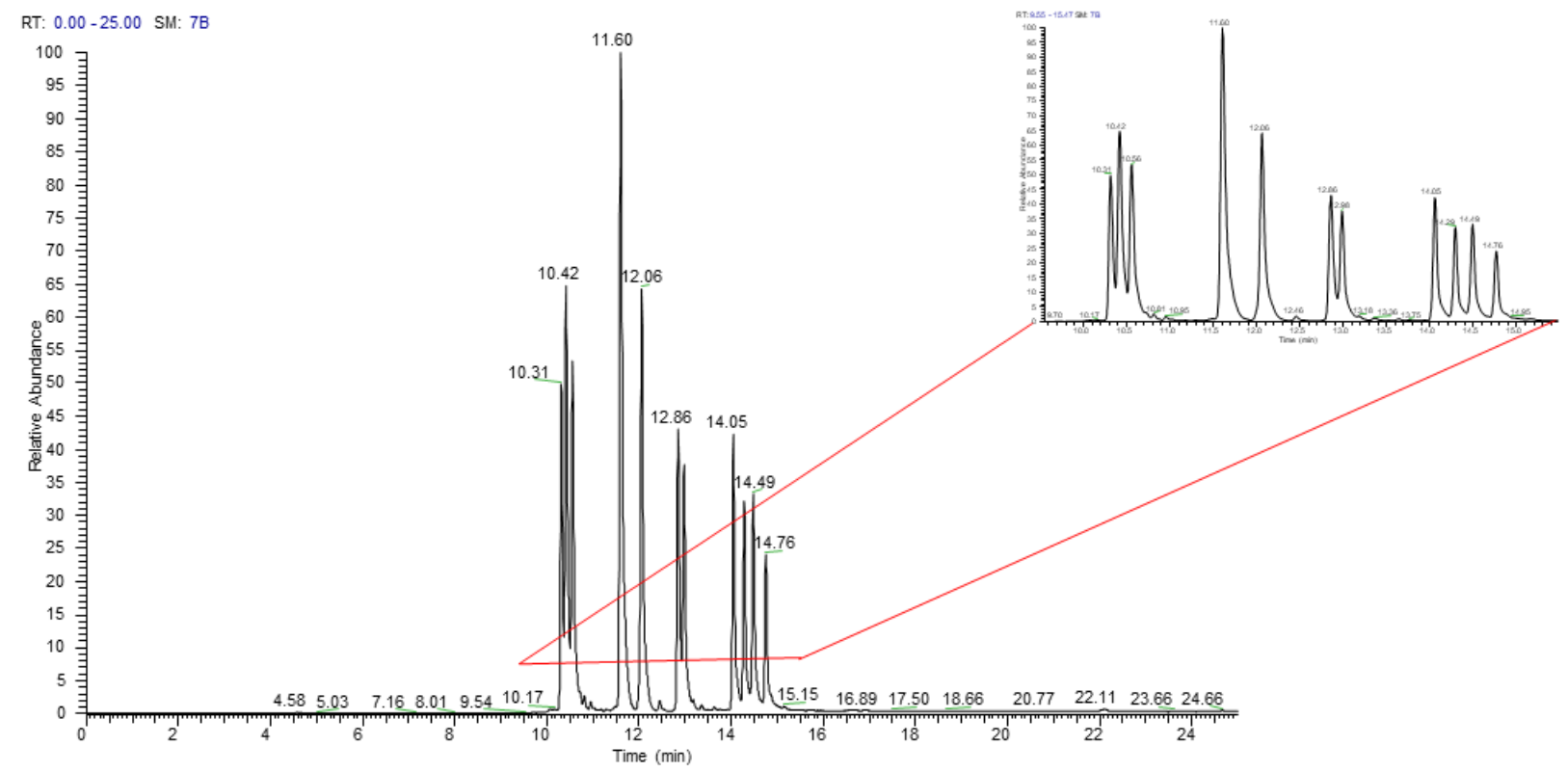

Characterisation of the monomer library. Total ion chromatogram of the initial foldamer library containing 12 different glutathione-protected monomers. Conditions of the analytical HPLC-MS measurement: Column: Aeris Widepore XB-C18 $(250 \times 4.6 \mathrm{~mm})$ Method: 5-80\% B during 25 minutes, flow rate: $0.7 \mathrm{~mL} \mathrm{~min}^{-1}$, where eluent $\mathrm{A}: 0.1 \% \mathrm{HCOOH}$ in water, eluent $\mathrm{B}: 0.1 \% \mathrm{HCOOH}$ in ACN. For retention time and molar mass of the compounds, see Supplementary Table 3.

\section{References}

1. Dinesh Gupta, A.R.K. Reactions of thiyl radicals. XIII. Photochemically induced exchange reactions of liquid alkyl disulfides. Can. J. Chem. 58, 1350-1354 (1980).

2. von Kiedrowski, G. Minimal Replicator Theory I: Parabolic Versus Exponential Growth, in Bioorganic Chemistry Frontiers. (eds. H. Dugas \& F.P. Schmidtchen) 113-146 (Springer Berlin Heidelberg, Berlin, Heidelberg; 1993).

3. Higgs, P.G. Chemical Evolution and the Evolutionary Definition of Life. J. Mol. Evol. 84, 225-235 (2017).

4. Bartus, É. et al. De Novo Modular Development of a Foldameric Protein-Protein Interaction Inhibitor for Separate Hot Spots: A Dynamic Covalent Assembly Approach. ChemistryOpen 6, 236-241 (2017). 